\title{
hsa_circ_0013401 Accelerates the Growth and Metastasis and Prevents Apoptosis and Autophagy of Neuroblastoma Cells by Sponging miR-195 to Release PAK2
}

\author{
Shibo Zhu $\mathbb{D}^{\text {, }}$, Xiangliang Tang $(\mathbb{D}$, Xiaofeng Gao $\mathbb{D}$, Jingqi Zhang $\mathbb{D}$, Yanhong Cui $\mathbb{D}$,

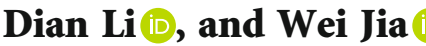 \\ Department of Pediatric Urology, Guangzhou Women and Children's Medical Center, Guangzhou Medical University, Guangzhou, \\ 510623 Guangdong, China \\ Correspondence should be addressed to Wei Jia; jiawei198044@hotmail.com
}

Received 2 April 2021; Revised 12 October 2021; Accepted 27 October 2021; Published 22 November 2021

Academic Editor: Fabio Ciccarone

Copyright (c) 2021 Shibo Zhu et al. This is an open access article distributed under the Creative Commons Attribution License, which permits unrestricted use, distribution, and reproduction in any medium, provided the original work is properly cited.

Background. Increased levels of circRNAs have been identified in a variety of cancers. However, the specific functions and mechanisms of circRNAs in neuroblastoma (NB) have not been fully explored. Methods. The levels of hsa_circ_0045997, hsa_circ_0080307, hsa_circ_0013401, hsa_circ_0077578, and microRNA-195 were confirmed by RT-qPCR in NB. Gainand loss-of-function assays and rescue experiments were conducted to determine the influence of hsa_circ_0013401, miR195, and P21-activated kinase 2 (PAK2) on the proliferation, apoptosis, autophagy, migration, and invasion of NB cells. Regulatory gene targets were validated by the luciferase assay. A xenograft mouse model was used to determine the in vivo effects of hsa_circ_0013401. Results. hsa_circ_0013401 was highly expressed, miR-195 was lowly expressed, and there was a negative correlation between hsa_circ_0013401 and miR-195 in NB. The inhibitory effects of hsa_circ_0013401 knockdown suppressed the proliferation, migration, and invasion and induced the apoptosis and autophagy of NB cells by targeting miR-195 to downregulate PAK2 expression. Luciferase reporter assays showed that miR-195 was a direct target of hsa circ_0013401, and PAK2 was the downstream target gene of miR-195. In vivo studies showed that hsa_circ_0013401 promotes tumor formation. Conclusions. hsa_circ_0013401 induced NB progression through miR-195 to enhance PAK2. Therefore, we might highlight a novel regulatory axis (hsa_circ_0013401/miR-195/PAK2) in NB.

\section{Introduction}

Neuroblastoma (NB) is a malignant tumor commonly discovered in the peripheral nervous system of infants [1]. NB is also a common embryonal solid tumor originating from the neural crests of the sympathetic nervous system [2]. Statistics show that the incidence of NB is second only to those of leukemia and brain cancer, and the mortality rate of children with NB is $\sim 15 \%$ [3]. Most NB patients are diagnosed with middle or late-stage disease due to the high degree of malignancy, rapid development, and easy metastasis of $\mathrm{NB}$ [4]. The current methods for treating NB are surgery, radiotherapy, chemotherapy, stem cell transplantation to enhance the effects of chemotherapy, and an induction of cell differentiation [5]. Despite combination therapy, the survival rate of patients with advanced NB remains low [6]. Therefore, there is an urgent need to identify specific molecules that can be exploited in targeted therapy regimens for NB patients.

Circular RNAs (circRNAs) comprise a class of noncoding RNA molecules without a cap structure at the $5^{\prime}$ ends and have a poly(A) tail at the $3^{\prime}$ ends, which can form a circular structure with covalent bonds [7]. Due to their closed ring structure, circRNAs are stable in cells and not easily degraded by exonuclease [8]. Moreover, circRNAs have developmental stage specificity and tissue specificity and are abundant in cells and highly conserved in different species [9]. Recent studies have shown that circRNAs have significant effects in regulating gene expression [10]. Several studies have demonstrated that circRNAs play critical roles in a variety of cancers, such as circHIPK3 in colorectal 
cancer [11], circRNA_102171 in thyroid cancer [12], circSLC8A1 in bladder cancer [13], and circCDR1 in gastric cancer [14]. Therefore, circRNAs can serve as diagnostic markers and therapeutic targets for tumors [15]. However, the role played by circRNA in NB development has been rarely investigated, and the biological functions and potential mechanisms of most circRNAs have yet to be discovered.

MicroRNAs (miRNAs), as crucial posttranscriptional regulators, can directly bind to the nontranslational sites of mRNAs via complementary base pairing and thus suppress protein translation or induce mRNA degradation [16, 17]. Recent studies have shown that circRNAs are rich in miRNA reaction elements (MREs) and can reduce the inhibitory effects of miRNAs on target gene expression by sponging miRNAs $[7,18]$. Compared with other types of competitive endogenous RNA (ceRNAs), circRNAs contain more binding sites for miRNA and therefore compete with endogenous RNA (ceRNA) [18]. Previous studies have shown that microRNA-195 (miR-195) is expressed at low levels in cancers [19-21], indicating that a low level of miR-195 expression may be related to cancer progression. Besides, recent research also suggested that miR-195 is lowly expressed in NB and miR-195 also can prevent the pyroptosis of NB cells [22]. However, the related circRNAs that can regulate miR-195 expression have been poorly reported in NB. We performed a bioinformatics analysis that revealed the existence of interaction sites between various circRNAs (has_circ_0045997, hsa_circ_0080307, has_circ_0013401, and hsa_circ_0077578) and miR-195. Therefore, we further investigated whether the potential functions and mechanisms of those circRNAs and miR-195 might significantly affect NB treatment.

In our study, we examined the expression levels of 4 circRNAs in NB tissues and discovered that hsa_circ_0013401 was highly expressed in NB. We then verified the targeted regulatory relationship between hsa_circ_0013401 and miR-195 and investigated the effects of hsa_circ_0013401 and miR-195 on the proliferation, apoptosis, autophagy, migration, and invasion of NB cells. Moreover, we confirmed that P21-activated kinase 2 (PAK2) was a target gene of miR-195 and proved the crucial roles played by the hsa_circ_0013401/miR-195/PAK2 axis in NB development and progression.

\section{Methods}

2.1. Tissue Samples. Samples of gangliocytoma tissue (GN, $n=8)$ and NB tissue $(n=8)$ were collected from patients who were diagnosed at the Guangzhou Women and Children's Medical Center of Guangzhou Medical University (Guangzhou, China). All histopathology assessments were performed by two professional pathologists using a doubleblind method. We also provided some basic information regarding the $8 \mathrm{GN}$ and $8 \mathrm{NB}$ patients (Table 1 ). All clinical studies were conducted in accordance with the Declaration of Helsinki, and each patient provided their written informed consent for study participation. The study protocol was approved by the Ethics Committee of Guangzhou
TABLE 1: Basic information for the $8 \mathrm{GN}$ and $8 \mathrm{NB}$ patients.

\begin{tabular}{lccccc}
\hline Patient ID & & Age & Gender & Location & NSE \\
\hline 1 & GN & 7 years & Female & Adrenal gland & 27.98 \\
2 & GN & 7 years & Female & Abdomen & 14.67 \\
3 & GN & 16 years & Female & Pelvic cavity & 13.12 \\
4 & GN & 5 years & Male & Abdomen & 30.38 \\
5 & GN & 6 years & Male & Abdomen & 16.87 \\
6 & GN & 4 years & Male & Abdomen & 23.41 \\
7 & GN & 10 years & Male & Pelvic cavity & 26.43 \\
8 & GN & 11 years & Male & Adrenal gland & 37.28 \\
9 & NB & 3 years & Male & Adrenal gland & $>370$ \\
10 & NB & 1 year & Female & Adrenal gland & 50.36 \\
11 & NB & 3 years & Male & Adrenal gland & 42.17 \\
12 & NB & 2 years & Male & Abdomen & $>370$ \\
13 & NB & 5 years & Male & Abdomen & 58.69 \\
14 & NB & 3 months & Female & Adrenal gland & 47.5 \\
15 & NB & 10 months & Female & Adrenal gland & 34.12 \\
16 & NB & 11 months & Female & Abdomen & $>370$ \\
\hline
\end{tabular}

Women and Children's Medical Center of Guangzhou Medical University (Guangzhou, China).

2.2. Cell Lines. Six NB cell lines (SK-N-BE, GNP, SH-SY5Y, IMR-32, LAN-1, and SK-N-SH) were provided by the Type Culture Collection of the Chinese Academy of Sciences (Shanghai, China). The cells were cultured in RPMI-1640 medium (HyClone Laboratories Inc., Logan, Utah, USA) containing 10\% fetal bovine serum (FBS, Life Technologies, cat. no. 10270) and 1\% penicillin/streptomycin (Gibco, Gaithersburg, $\mathrm{MD}, \mathrm{USA}$ ) at $37^{\circ} \mathrm{C}$ in an incubator with $5 \% \mathrm{CO}_{2}$.

2.3. Fluorescence In Situ Hybridization (FISH). The samples were added to Carnoy's solution and then centrifuged at $1000 \mathrm{~g}$ for 6 minutes. Following centrifugation, $100 \mu \mathrm{L}$ aliquots of solution were placed onto clean glass slides. Prehybridization was performed by treatment with a hybridization solution (WAKO, cat. no. \#544-01331) for 1 hour (h) at $37^{\circ} \mathrm{C}$; after which, probes $(100 \mathrm{nM})$ were added to the slides. After denaturation at $74^{\circ} \mathrm{C}$ for $6 \mathrm{~min}$, the slides were incubated overnight in a humid chamber at $35^{\circ} \mathrm{C}$ and then washed again with $0.4 \times \mathrm{SSC}$ at $45^{\circ} \mathrm{C}$. The slides were then treated with $2 \times$ SSC containing $0.05 \%$ Tween-20 at room temperature (RT) for $1 \mathrm{~min}$. After nuclear staining with DAPI (Life Science), the results were visualized with a fluorescence microscope.

2.4. Immunohistochemistry (IHC) Assay. GN and NB tissues were soaked in $10 \%$ paraformaldehyde (Sigma, St. Louis, $\mathrm{MO}, \mathrm{USA}$ ) at $4^{\circ} \mathrm{C}$ for $12 \mathrm{~h}$ and then dehydrated. After embedding, a rotary microtome (Leica, GER) was used to cut the tissue samples into $3.5 \mu \mathrm{m}$ thick sections. The sections were immersed in a $42.5^{\circ} \mathrm{C}$ water bath and then mounted onto microscope slides (Citoglas, China). After deparaffinization and rehydration, the tissue sections were processed using reagents in an SABC kit (Bosterbio, China) 
TABle 2: Primer sequences used in the RT-qPCR assay.

\begin{tabular}{lc}
\hline Name & Sequence $\left(5^{\prime}-3^{\prime}\right)$ \\
\hline GAPDH forward & TGTTCGTCATGGGTGTGAAC \\
GAPDH reverse & ATGGCATGGACTGTGGTCAT \\
PAK2 forward & CACCCGCAGTAGTGACAGAG \\
PAK2 reverse & GGGTCAATTACAGACCGTGTG \\
U6 forward & CTCGCTTCGGCAGCACA \\
U6 reverse & AACGCTTCACGAATTTGCGT \\
hsa-miR-195-5p forward & CTCAACTGGTGTCGTGGAGTCGGCAATTCAGTTGAGGCCAATAT \\
hsa-miR-195-5p reverse & ACACTCCAGCTGGGTAGCAGCACAGAAATAT \\
hsa_circ_0013401 forward & GTCCTGACTTGTCATGTGCTG \\
hsa_circ_0013401 reverse & CAGACATTCACAAAGGAGCAA \\
hsa_circ_0080307 forward & TGCTGCTAAAACCTGTCCAAC \\
hsa_circ_0080307 reverse & CCACAGCAGCAATACGAACC \\
hsa_circ_0077578 forward & TGGATGAGATGCCGGTCAA \\
hsa_circ_0077578 reverse & TAAAGCATGCATCTGTGCGT \\
hsa_circ_0045997 forward & CTGCGTTTGGAGCCGTT \\
hsa_circ_0045997 reverse & CAGACCAGCAGTCAGAGCGT \\
\hline
\end{tabular}

according to instructions provided by the manufacturer. After processing, the mounted sections were incubated with anti-Ki67 (1:200, Abcam, UK) and anti-PAK2 (1:100, Abcam, UK) antibodies at $4^{\circ} \mathrm{C}$ for $12 \mathrm{~h}$ and then stained with reagents in a DAB kit (Bosterbio, China). The staining results were obtained using an inverted microscope (Nikon Eclipse TI-SR, Japan).

2.5. RNA Transfection. miR-195 mimics, miR-195 inhibitor, and a negative control (NC) were all designed and obtained from GenePharma (China). circ_0013401-overexpression plasmids, circ_0013401 shRNA, and PAK2-overexpression plasmids were obtained from BioVector (Newark, CA, USA). All cell transfections were performed using Lipofectamine 3000 (Invitrogen, Carlsbad, CA, USA) according to the manufacturer's instructions.

2.6. Quantitative Real-Time PCR (RT-PCR). The total RNA was extracted from NB tissue samples and treated NB cells using the TRIzol reagent (Invitrogen). After inspection, the RNA extracts were reverse transcribed to cDNA using a Bes$\operatorname{tar}^{\mathrm{TM}}$ qPCR RT kit (DBI Bioscience, cat. no. \#2220). RT-PCR was performed using Bestar ${ }^{\mathrm{TM}}$ qPCR MasterMix (DBI Bioscience, cat. no. \#2043). The sequences of the primers used in this study are shown in Table 2 . The levels of gene expression were calculated using the $2^{-\Delta \Delta \mathrm{Ct}}$ method.

2.7. Cell Counting Kit-8 (CCK-8) Assay. The CCK-8 assay (Dojindo, Rockville, MD, USA) was used to evaluate the viability of treated NB cells. In brief, the treated NB cells were seeded into 96 -well plates $\left(2 \times 10^{4}\right.$ cells/well $)$ and cultured at $37^{\circ} \mathrm{C}$ for $24 \mathrm{~h}$. Next, $10 \mu \mathrm{L}$ of CCK- 8 solution was added to each well, and these plates were incubated at $37^{\circ} \mathrm{C}$ for $10 \mathrm{~min}$. The optical density (OD) of each well was determined at $450 \mathrm{~nm}$.
2.8. EdU Assay. Treated SH-SY5Y and SK-N-BE cells were fixed with $0.5 \%$ paraformaldehyde (Sigma) and then treated with EdU solution (RioBio, Guangzhou, China) for $30 \mathrm{~min}$. Subsequently, a flow cytometer (Becton Dickinson, Franklin Lakes, NJ, USA) was used to assess cell proliferation.

2.9. Colony Formation Assay. Briefly, the SH-SY5Y and SK$\mathrm{N}$-BE cells in each group were plated into 6-well plates (2000 cells/well) and cultured at $37^{\circ} \mathrm{C}$ for 14 days. The cells were then fixed with $4 \%$ paraformaldehyde and stained with Giemsa solution. The numbers of cell colonies were counted under a microscope.

2.10. Transwell Assay. The invasion or migration abilities of $\mathrm{SH}-\mathrm{SY} 5 \mathrm{Y}$ and SK-N-BE cells in each group were assessed using Transwell chambers $(8 \mu \mathrm{m}$, Corning, Corning, NY, USA) with or without a Matrigel coating, respectively. Cells $\left(1 \times 10^{6}\right.$ cells $)$ suspended in $500 \mu \mathrm{L}$ of serum-free medium were seeded into the upper chamber, and $500 \mu \mathrm{L}$ of culture medium containing $15 \%$ FBS was added to the lower chamber. After $24 \mathrm{~h}$ of incubation, the invaded or migrated cells were fixed with $4 \%$ paraformaldehyde (Sigma) and stained with $5 \%$ crystal violet solution (Sigma). The numbers of invaded or migrated cells were counted under a microscope.

2.11. Flow Cytometric Analysis. The apoptosis rates of $\mathrm{SH}-$ SY5Y and SK-N-BE cells in each group were monitored by flow cytometry performed using the Annexin V/FITC double staining method (BD Biosciences) according to instructions.

2.12. Transmission Electron Microscopy (TEM). After washing, the treated SH-SY5Y and SK-N-BE cells were fixed in $2 \%$ glutaraldehyde for $2 \mathrm{~h}$, treated with $1 \%$ osmium tetroxide for $1 \mathrm{~h}$, and then dehydrated using a graded ethanol series for $2 \mathrm{~h}$. TEM observations were carried out using a Leo 912 $\mathrm{AB}$ electron microscope. 

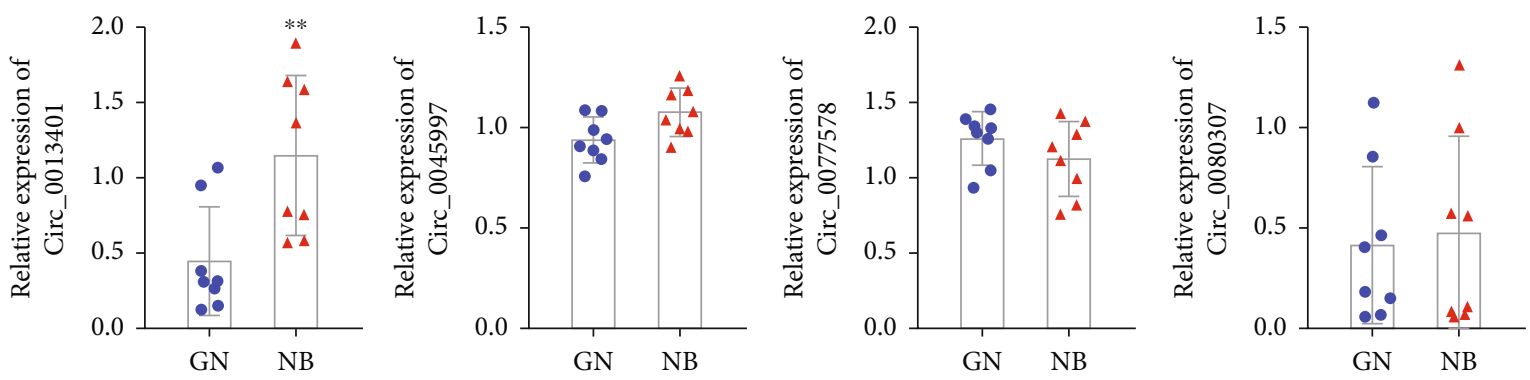

(a)
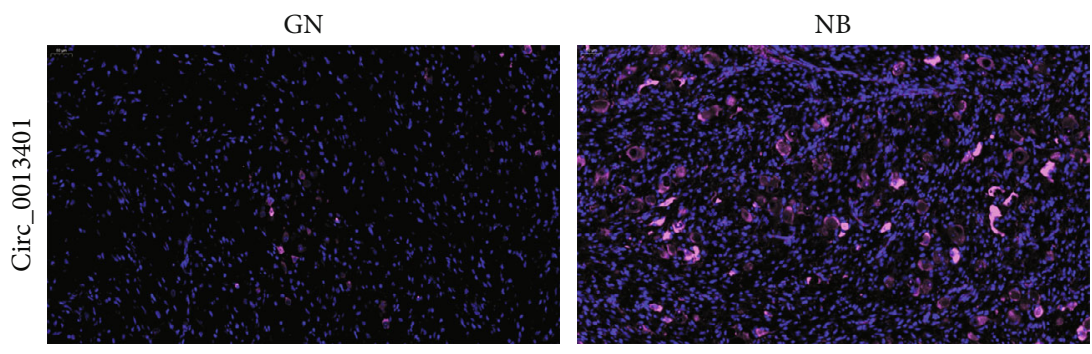

(b)

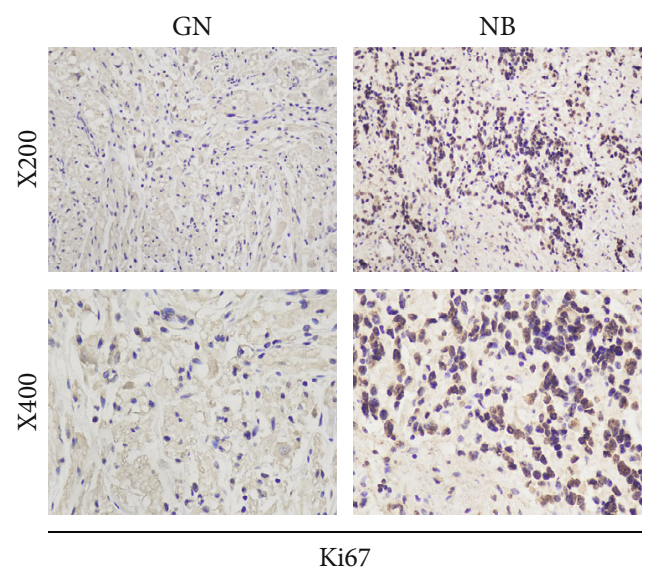

(c)

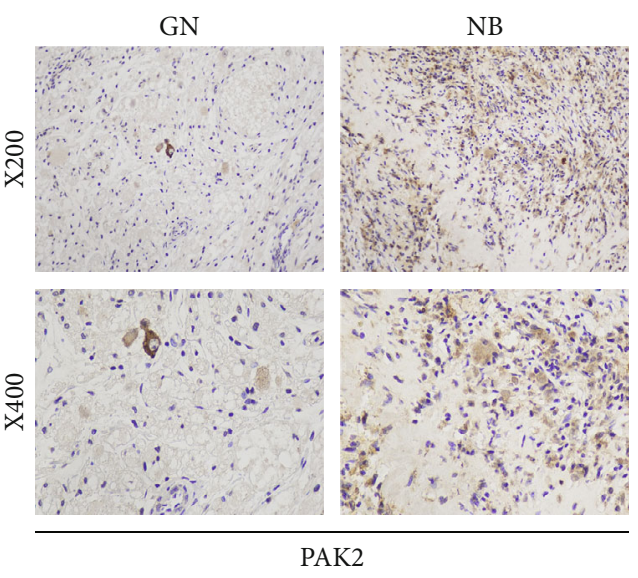

(d)

Figure 1: circ_0013401 was highly expressed in NB. (a) The levels of circ_0013401, circ_0045997, circ_0077578, and circ_0080307 expression in samples of GN and NB tissues were confirmed ( $n=8$ tissues per group). (b) circ_0013401 expression in GN and NB tissues was examined by FISH. Magnification, $\times 200$. IHC assays were performed to examine the expression of Ki67 (c) and PAK2 (d) in GN and NB tissues. Magnification, $\times 200$; magnification, $\times 400 .{ }^{* *} p<0.01$.

2.13. Immunofluorescence Staining. After fixation, the treated SH-SY5Y and SK-N-BE cells were incubated with $5 \%$ Tween-20 for $2 \mathrm{~h}$. The cells were then blocked with $10 \%$ normal goat serum for $1 \mathrm{~h}$ and subsequently incubated overnight with anti-LC3 (1:100, Abcam, cat. no. ab62720) and anti-PAK2 (Abcam, cat. no. ab3442) primary antibodies, followed by incubation with a secondary antibody (Abcam, cat. no. DAR-546) for $2 \mathrm{~h}$. The cell nucleus was stained with DAPI (Life Science) for $30 \mathrm{~min}$, and results were photographed using a confocal laser microscope (Zeiss, Germany, LSM710).

2.14. Western Blotting. The total proteins were extracted using RIPA buffer (Beyotime, Shanghai, China), and the protein concentration in each extract was detected using a BCA kit (Beyotime, China). A $50 \mu \mathrm{g}$ sample of protein from each extract was separated by SDS-PAGE (10\%), and the protein bands were transferred onto PVDF membranes (Millipore, Burlington, MA, USA), which were subsequently blocked with $5 \%$ nonfat milk for $2 \mathrm{~h}$. Next, the membranes were incubated overnight with primary antibodies, followed by incubation with an HRP-conjugated secondary antibody for $1.5 \mathrm{~h}$. Signals were detected by using enhanced chemiluminescence (ECL, Thermo Fisher Scientific, Waltham, MA, USA) and analyzed using ImageJ software.

2.15. In Vivo Tumor Growth Assay. Male BALB/c nude mice ( 8 weeks old) were provided by the Laboratory Animal Center of Southern Medical University (no. 44002100023925). The protocol for the in vivo study was approved by the Institutional Animal Ethics Committee of Guangzhou Medical University (Guangzhou, China), and the study was carried 


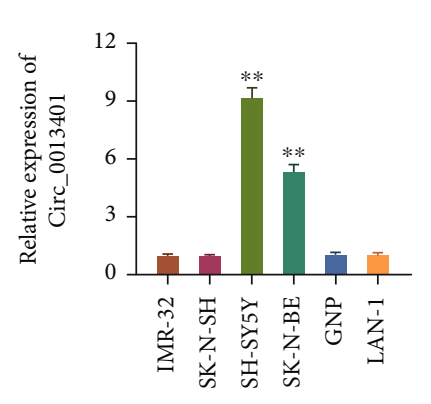

(a)

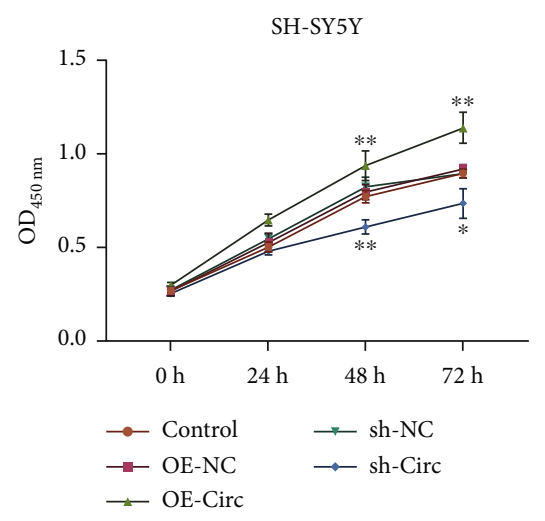

SH-SY5Y

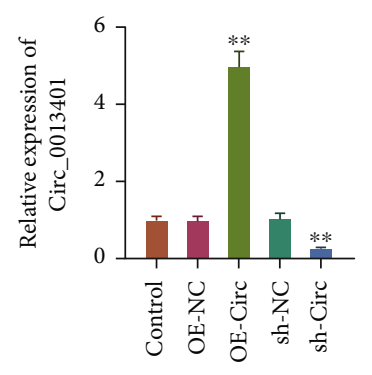

(b)

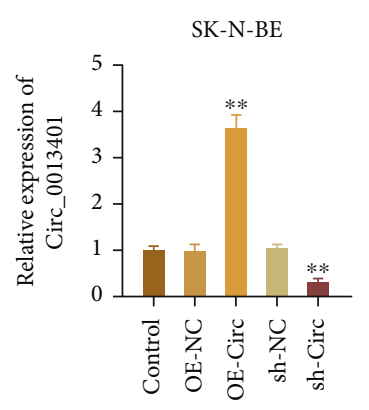

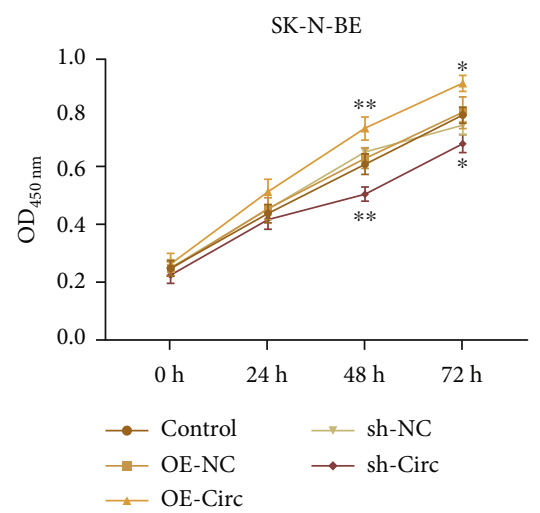

(c)
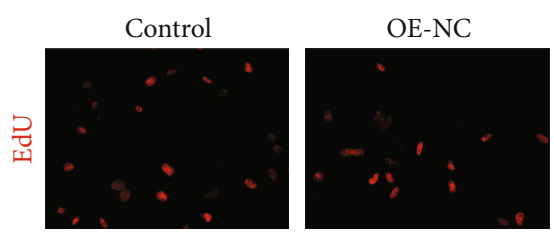

OE-Circ

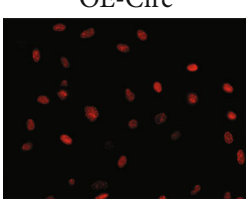

sh-NC
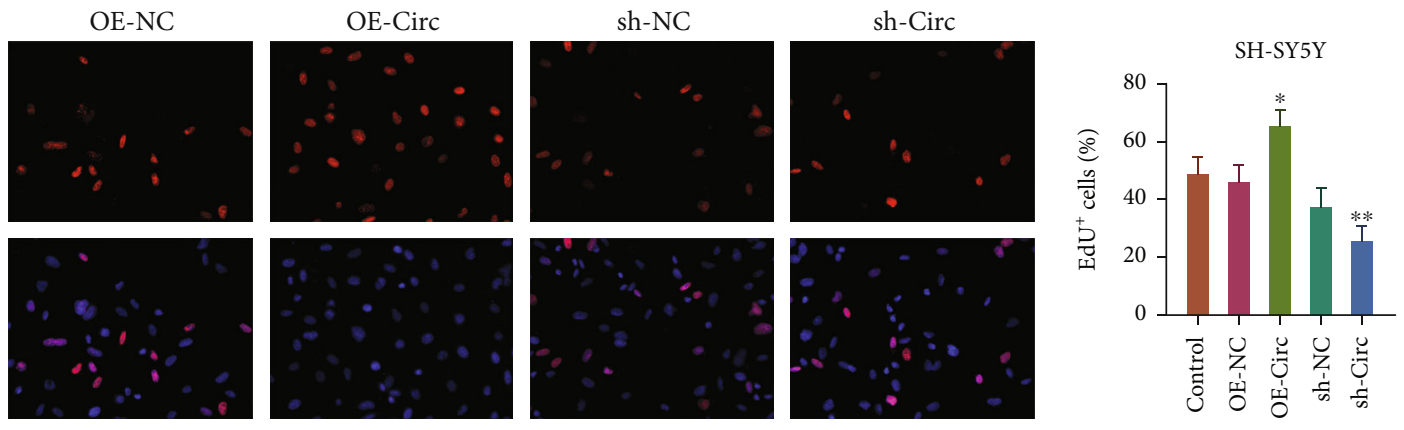

Control

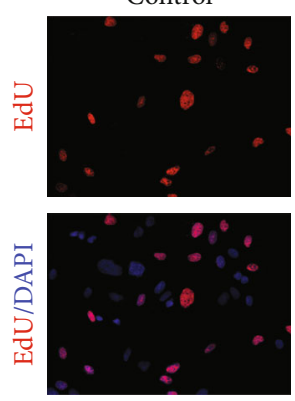

OE-NC
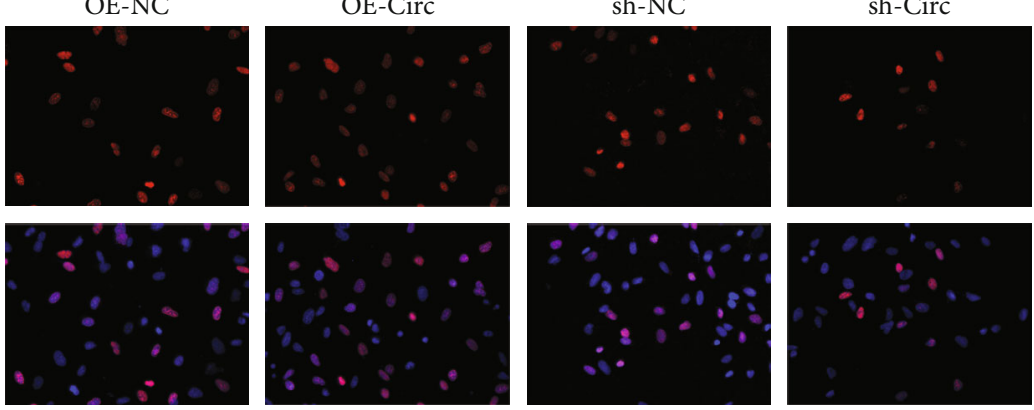

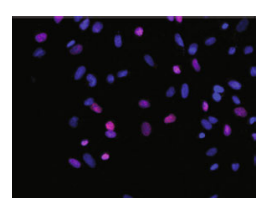

(d)

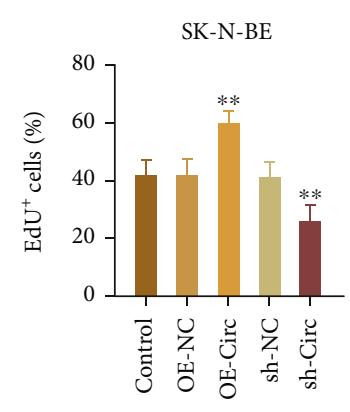

FIgURE 2: Continued. 

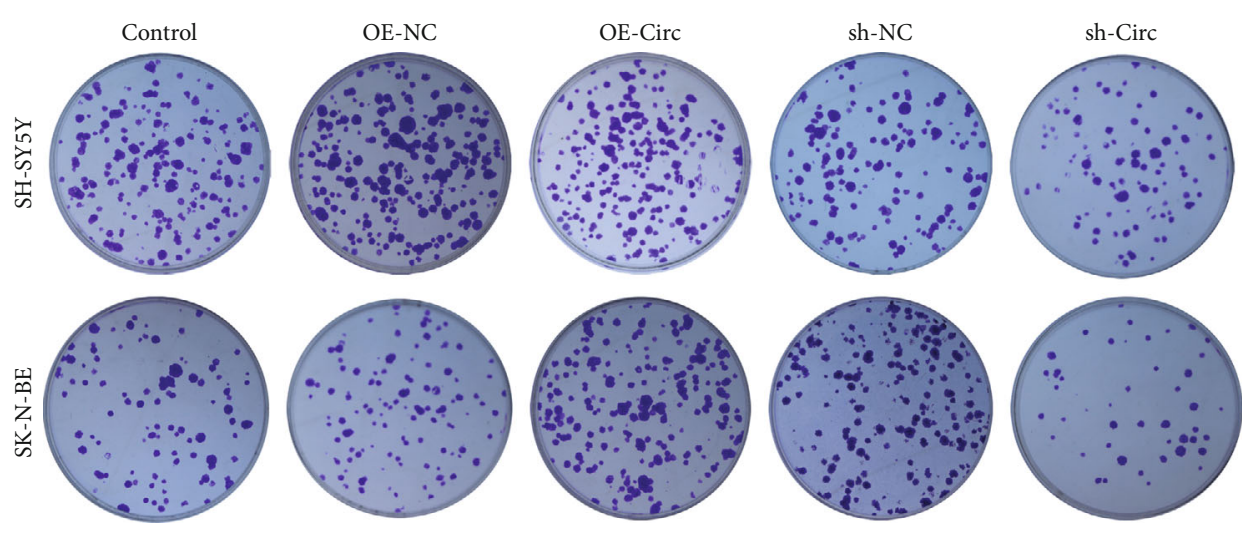
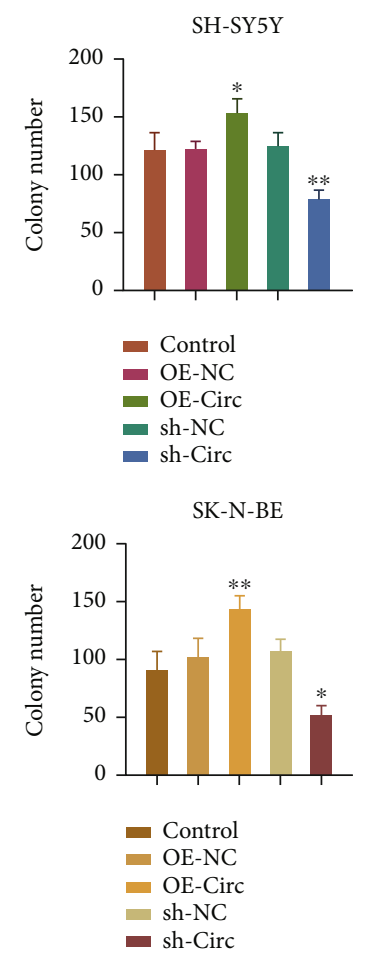

(e)

Figure 2: circ_0013401, as an oncogene, significantly increased NB cell proliferation. (a) circ_0013401 expression in different neuroblastoma cell lines (SK-N-BE, GNP, SH-SY5Y, IMR-32, LAN-1, and SK-N-SH) was determined by RT-qPCR. (b) SH-SY5Y and SK-N-BE cells were transfected with a circ_0013401-overexpression plasmid or circ_0013401 shRNA, and the resultant effects were examined using RT-qPCR assays. (c) CCK-8 assays were performed to confirm the effect of circ_0013401 overexpression or knockdown on the proliferation of SH-SY5Y and SK-N-BE cells. Cell proliferation was also assessed by EdU staining (d) and the clone formation assay (e) in SH-SY5Y and SK-N-BE cells with circ_0013401 overexpression or silencing. ${ }^{*} p<0.05,{ }^{* *} p<0.01$.

out at the laboratory animal center. Briefly, transfected SH-SY5Y cells $\left(200 \mu \mathrm{L} ; 1 \times 10^{7}\right.$ cells) were subcutaneously injected into the left flank of nude mice; after which, tumor size was measured every 7 days up to 28 days after injection. After 28 days, the mice were sacrificed by anesthesia with sodium pentobarbital and the xenograft tumors were excised. The tumor volumes were calculated using the formula length $\times$ width $^{2} \times 0.5$.

2.16. Dual-Luciferase Reporter Assay. To verify the relationship between circ_0013401 and miR-195 in SH-SY5Y and SK-N-BE cells, circ_0013401-wild type (WT) and circ_ 0013401-mutant (Mut) plasmids were constructed using the WT and Mut fragments of circ_0013401, including the putative miR-195 binding sites and psiCheck-2 vector (Promega, cat. no. C8021). Next, SH-SY5Y or SK-N-BE cells were added to the wells of 6 -well plates $\left(1 \times 10^{4}\right.$ cells/well $)$ and incubated overnight at $37^{\circ} \mathrm{C}$. The cells were then cotransfected with miR-195 mimics plus the circ_0013401WT or circ_0013401-Mut. The firefly and Renilla luciferase activities of the SH-SY5Y and SK-N-BE cells in each group were examined using a Dual-Luciferase Assay System (Promega, Madison, WI, USA).

2.17. Statistical Analysis. All data were analyzed using IBM SPSS Statistics for Windows, version 20.0 software (IBM
Corp., Armonk, NY, USA). Results are expressed as the mean value \pm SEM of data obtained from three independent experiments. The significance of differences between groups was analyzed by Student's $t$-test or one-way analysis of variance. A $p$ value $<0.05$ was considered to be statistically significant.

\section{Results}

3.1. circ_0013401 Was Highly Expressed in NB. To investigate the changes that occurred in circRNA expression in $\mathrm{NB}$, we first examined the levels of circ_0013401, circ 0045997, circ_0077578, and circ_0080307 expression in 8 gangliocytoma (GN) and 8 neuroblastoma (NB) tissue samples. As shown in Figure 1(a), only circ_0013401 expression was significantly upregulated in the NB tissues when compared with the GN tissues $(p<0.01)$. Next, we performed a FISH assay to identify circ_0013401 expression in GN and NB tissues and found that circ_0013401 was much more highly expressed in the NB tissues than in the GN tissues (Figure 1(b)). Additionally, the levels of Ki67 and PAK2 expression were examined using IHC assays, and those results showed that expression of both Ki67 and PAK2 was markedly elevated in the NB tissues when compared with the GN tissues (Figures 1(c) and 1(d)). Consequently, we 


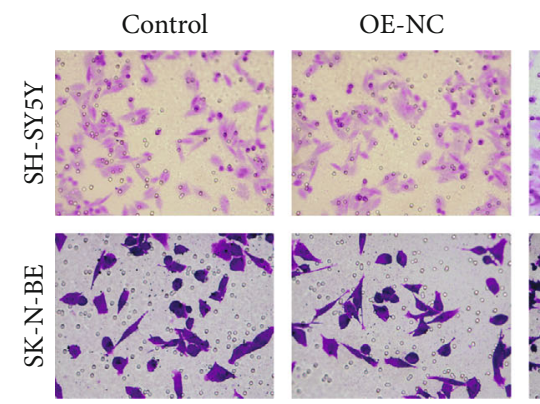

OE-Circ

sh-NC

sh-Circ
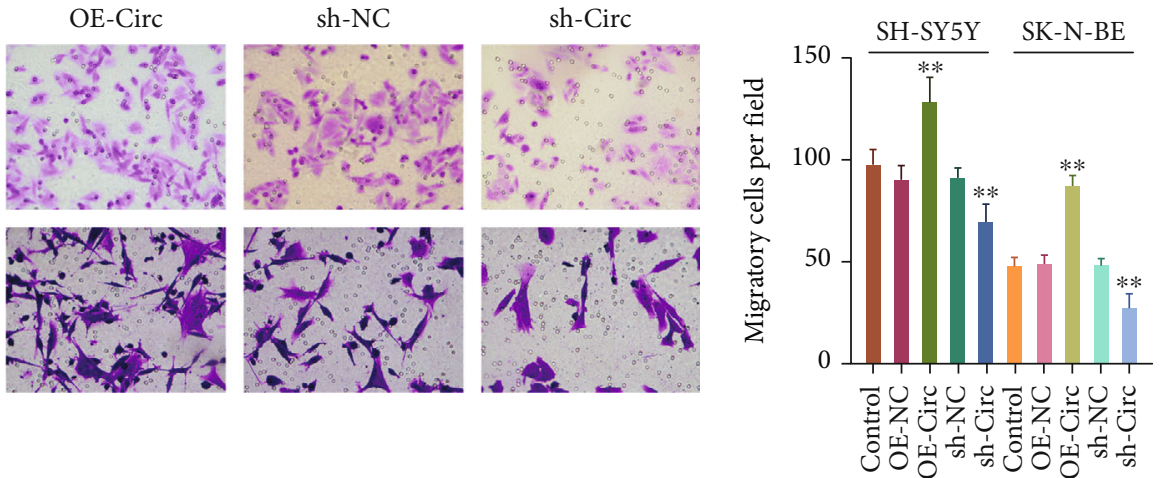

(a)

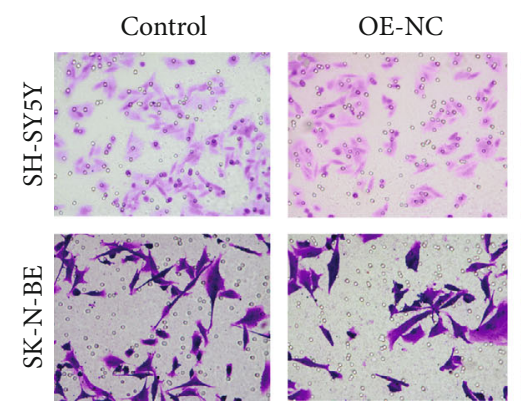

OE-Circ

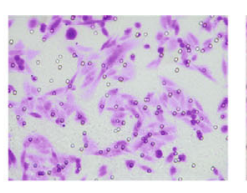

sh-NC

sh-Circ
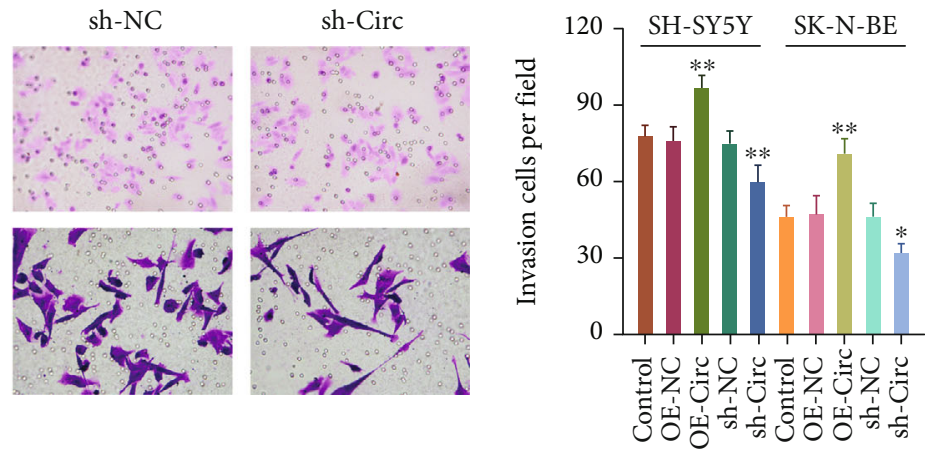

(b)
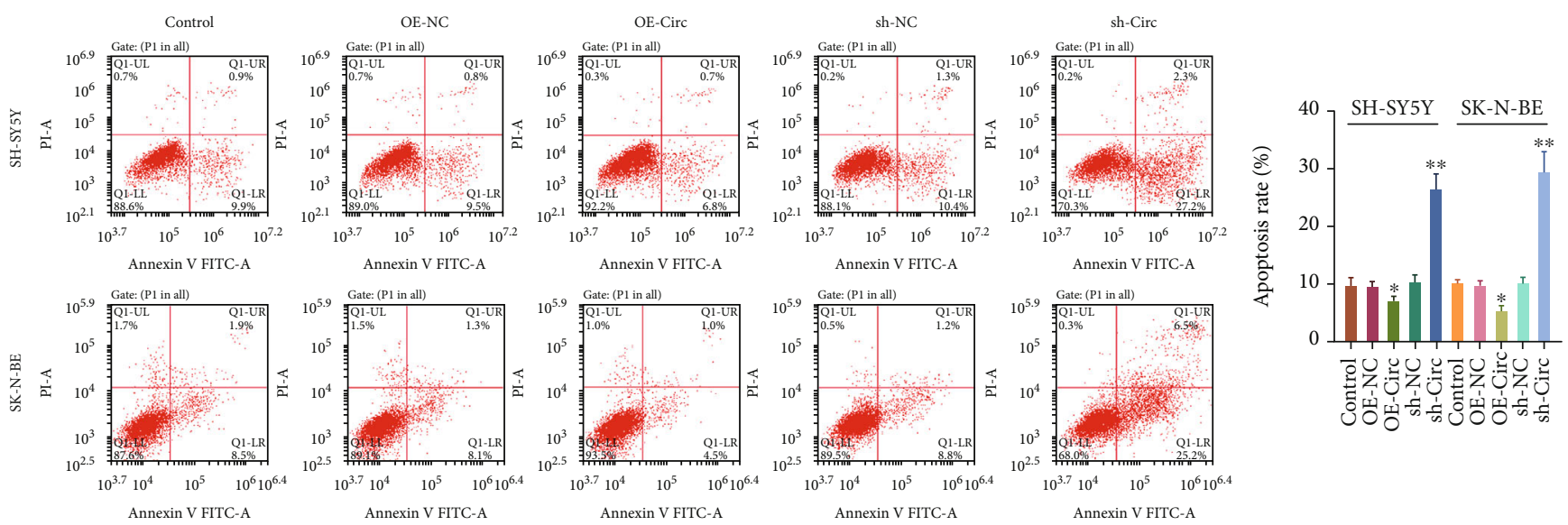

(c)

Figure 3: Continued. 

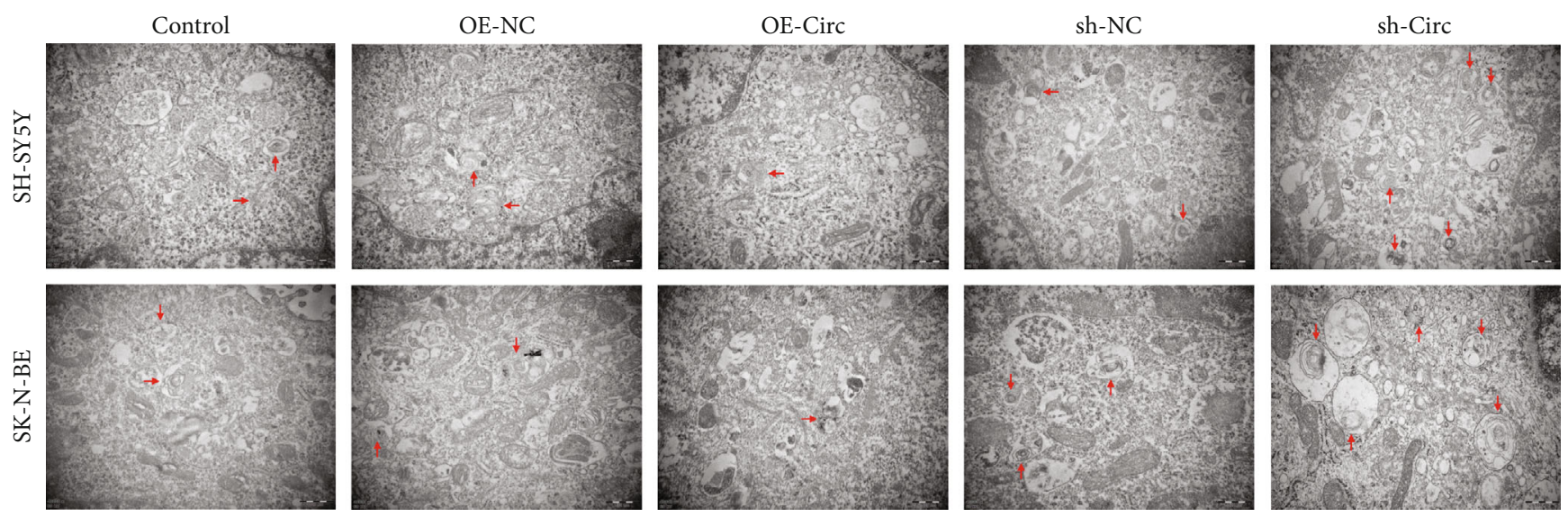

(d)

FIgURE 3: circ_0013401 markedly facilitated NB cell migration and invasion and prevented NB cell apoptosis and autophagy. Transwell assays were performed to identify the effects of circ_0013401 overexpression or knockdown on the migration (a) and invasion (b) capabilities of SH-SY5Y and SK-N-BE cells; the numbers of migrated and invading cells were counted. (c) The effects of circ_0013401 overexpression or knockdown on the apoptosis of SH-SY5Y and SK-N-BE cells were evaluated by flow cytometry, and the numbers of apoptotic cells in each group were calculated. (d) Changes in autophagy (red arrow) that occurred in SH-SY5Y and SK-N-BE cells with circ_0013401 overexpression or knockdown were examined by TEM. ${ }^{*} p<0.05,{ }^{* *} p<0.01$.

confirmed that circ_0013401, Ki67, and PAK2 were highly expressed in NB.

3.2. circ_0013401, as an Oncogene, Significantly Accelerated NB Cell Proliferation. Given that circ_0013401 was upregulated in NB tissues, we investigated the effects of circ 0013401 overexpression and knockdown on various functions in NB cells. We first examined the levels of circ_0013401 expression in different neuroblastoma cell lines (SK-N-BE, GNP, SH-SY5Y, IMR-32, LAN-1, and SK-N-SH) using RTqPCR and found that circ_0013401 expression was significantly upregulated in SH-SY5Y and SK-N-BE cells when compared with other NB cell lines. Therefore, SH-SY5Y and SK-N-BE cells were selected for use in subsequent experiments $(p<0.01$, Figure 2(a)). RT-qPCR analyses were performed to determine how transfection with a circ_0013401-overexpression plasmid or circ_0013401 shRNA might affect SH-SY5Y and SK-N-BE cells. As shown in Figure 2(b), circ_0013401 expression was upregulated in the circ_0013401 overexpression group when compared with the overexpression-NC group. Furthermore, circ_0013401 expression was significantly downregulated in the circ_0013401 knockdown group when compared with the sh-NC group $(p<0.01$, Figure 2(b)). Next, we examined how circ_0013401 overexpression or knockdown affected the proliferation of $\mathrm{SH}$ SY5Y and SK-N-BE cells. Results of CCK-8 assays showed that SH-SY5Y and SK-N-BE cells in the circ_0013401 overexpression groups were significantly more viable than those in the overexpression-NC group, while cell viability was dramatically decreased in the circ_0013401 knockdown groups relative to the sh-NC group $(p<0.05, p<0.01$, Figure 2(c)). Consequently, the numbers of $\mathrm{EdU}^{+}$in circ 0013401-overexpressing SH-SY5Y and SK-N-BE cells were significantly higher than those in the overexpression-NC group, and the numbers of EdU ${ }^{+}$circ_0013401-silenced cells were dramatically lower than those in the sh-NC group $(p<0.05, p<0.01$, Figure 2(d)). Meanwhile, clone formation assays revealed that circ_0013401 overexpression induced NB cell proliferation, and circ_0013401 knockdown suppressed NB cell proliferation $(p<0.05, p<0.01$, Figure 2(e)). These findings verified that circ_0013401 played a significant role in inducing the proliferation of NB cells.

3.3. circ_0013401 Facilitated the Migration and Invasion of NB Cells and Prevented Their Apoptosis and Autophagy. We next sought to further verify the changes in migration, invasion, apoptosis, and autophagy that occurred in $\mathrm{SH}$ SY5Y and SK-N-BE cells following circ_0013401 overexpression or knockdown. First, the migration and invasion capabilities of transfected SH-SY5Y and SK-N-BE cells were investigated using Transwell assays. The resultant data showed that overexpression of circ_0013401 enhanced the migration and invasion capabilities of the cells, while circ 0013401 knockdown led to significant reductions in SHSY5Y and SK-N-BE cell migration and invasion $(p<0.05$, $p<0.01$, Figures 3(a) and 3(b)). Data from flow cytometry studies indicated that circ_0013401 overexpression notably reduced the apoptosis rate of both cell lines, and circ 0013401 knockdown significantly increased the apoptosis rates of SH-SY5Y and SK-N-BE cells $(p<0.05, p<0.01$, Figure $3(\mathrm{c})$ ). Moreover, transmission electron microscopy (TEM) studies revealed that when compared to cells in the control groups, the numbers of autophagosomes in circ 0013401-overexpressing SH-SY5Y and SK-N-BE cells were significantly reduced, while large numbers of autophagosomes were seen in the circ_0013401-silenced SH-SY5Y and SK-N-BE cells (Figure 3(d)). These findings indicated that circ_0013401 could induce migration and invasion 


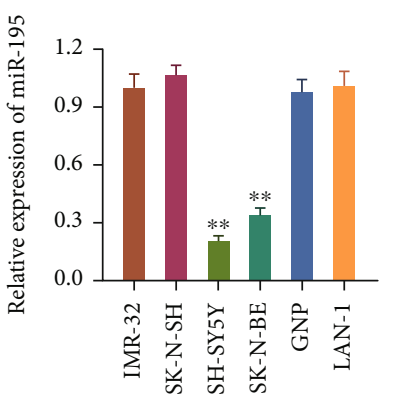

(a)
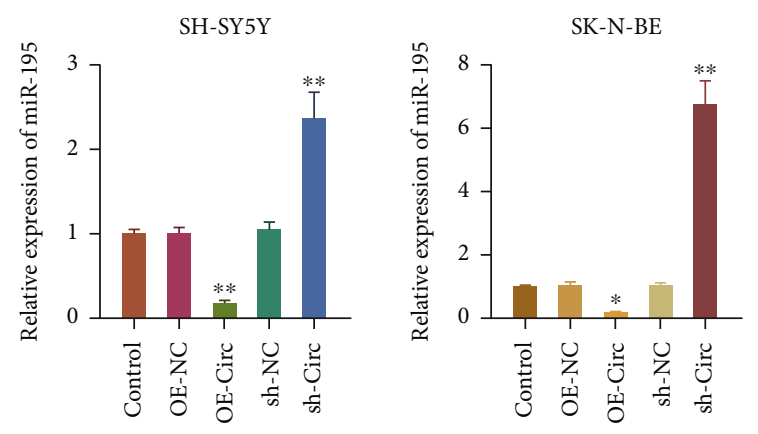

(c)
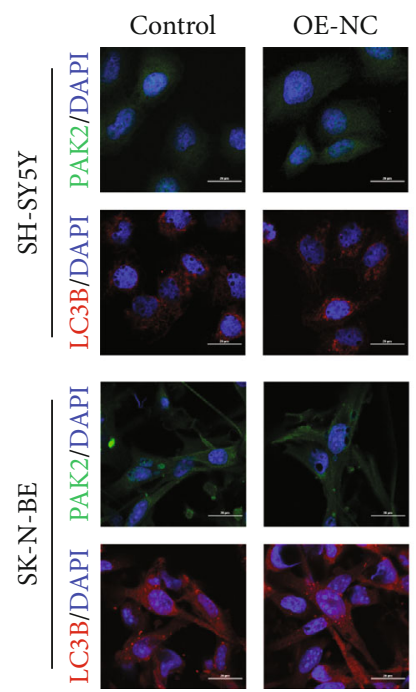

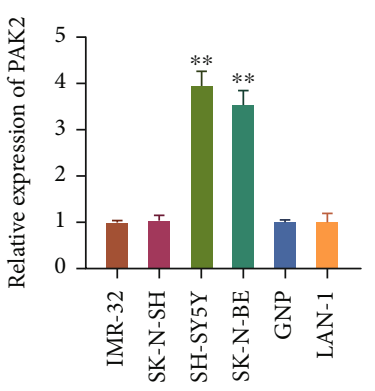

(b)

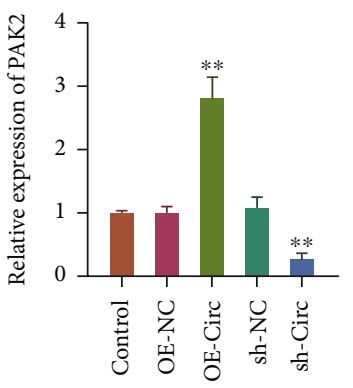

SK-N-BE

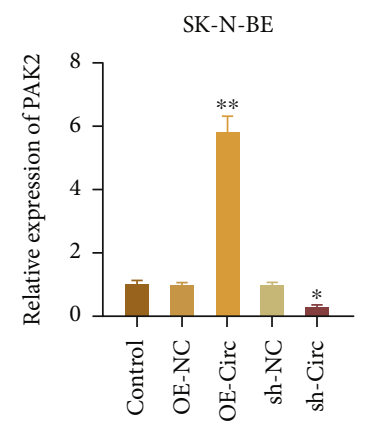

(d)

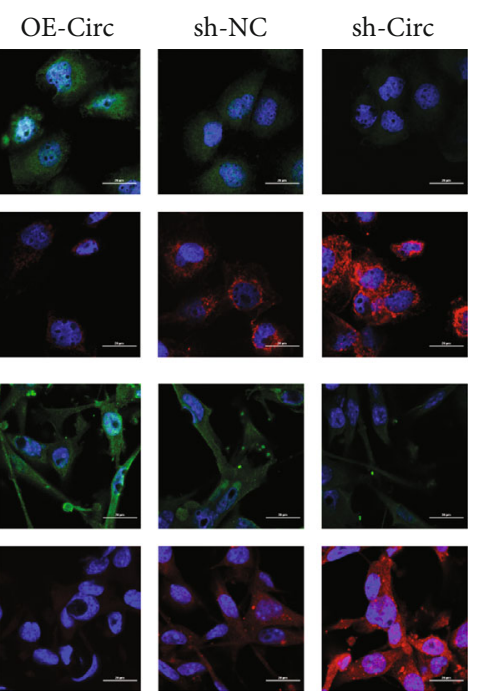

(e)

FIgURE 4: Continued. 

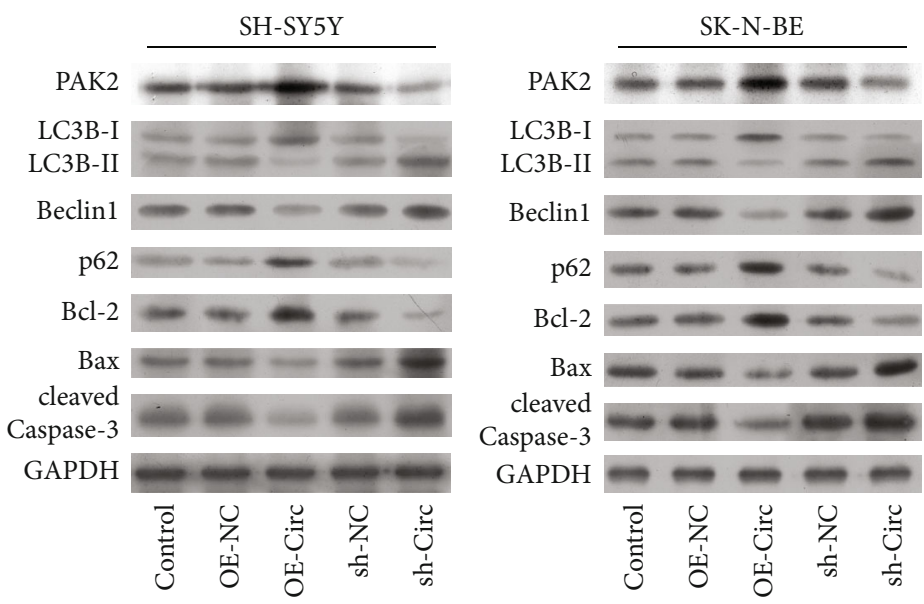

(f)

FIGURE 4: circ_0013401 regulated miR-195, PAK2, and autophagy- and apoptosis-related proteins in NB cells. (a) RT-qPCR analysis of miR195 expression in different NB cell lines (SK-N-BE, GNP, SH-SY5Y, IMR-32, LAN-1, and SK-N-SH). (b) PAK2 expression was tested using RT-qPCR in different neuroblastoma cell lines (SK-N-BE, GNP, SH-SY5Y, IMR-32, LAN-1, and SK-N-SH). (c) The effects of circ_0013401 overexpression or knockdown on miR-195 expression were assessed by RT-qPCR. (d) The changes in PAK2 expression that occurred in SHSY5Y and SK-N-BE cells with circ_0013401 overexpression or silencing were evaluated by RT-qPCR. (e) PAK2 and LC3B expression in SHSY5Y and SK-N-BE cells with circ_0013401 overexpression or knockdown was determined using IF assays. Magnification, $\times 400$; scale bar $=20 \mu \mathrm{m}$. (f) Western blotting was used to examine the levels of PAK2, LC3B, Beclin1, p62, Bcl-2, Bax, and cleaved caspase3 in SH-SY5Y and SK-N-BE cells with circ_0013401 overexpression or silencing. ${ }^{*} p<0.05,{ }^{* *} p<0.01$.

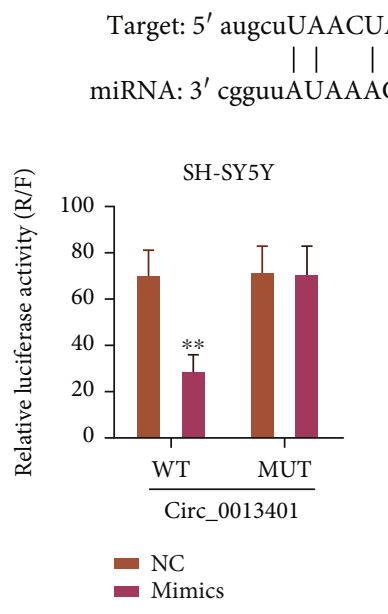

(a)

\author{
Target : $5^{\prime}$ gucuuccUGUCUCCGCUGCUc3' \\ | | || |||||| \\ miRNA : $3^{\prime}$ cgguuauAAAGACACGACGAu $5^{\prime}$
}
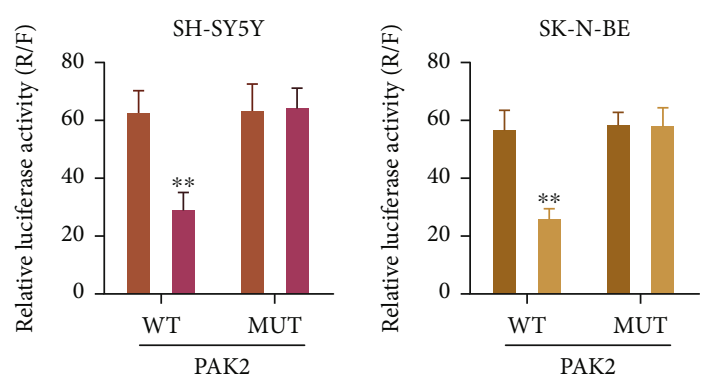

- $\mathrm{NC}$

(b)

FIGURE 5: circ_0013401 sponged miR-195, and PAK2 was a target gene of miR-195. (a) Dual-luciferase reporter assays were conducted to investigate the interplay between circ_0013401 and miR-195. (b) A dual-luciferase reporter assay proved that PAK2 was a direct target gene of miR-195.

and repress apoptosis and autophagy in SH-SY5Y and SK$\mathrm{N}-\mathrm{BE}$ cells.

3.4. circ_0013401 Regulated the miR-195/PAK2 Axis, as well as Autophagy- and Apoptosis-Related Proteins in NB Cells. Subsequently, we sought to identify the biological pathway that regulates circ_0013401 in NB. Bioinformatics predictions indicated that circ_0013401 might be a miRNA response element (MRE) of miR-195, which might bind with circ_0013401. Those predications also suggested PAK2 as the most likely target gene of miR-195. Therefore, miR-195 and PAK2 became the focus of our mechanistic studies. To further confirm the levels of miR-195 expression in NB, the levels of miR-195 expression in different neuroblastoma cell lines were analyzed using the RT-qPCR. As indicated in Figure 4(a), miR-195 expression was significantly downregulated in SH-SY5Y and SK-N-BE cells when compared with the other cell lines $(p<0.01)$. Meanwhile, we discovered that PAK2 expression was dramatically elevated in SH-SY5Y and SK-N-BE cells relative to the other cell lines $(p<0.01$, Figure 4(b)). In addition, we demonstrated that miR-195 could be downregulated by circ_0013401 overexpression 

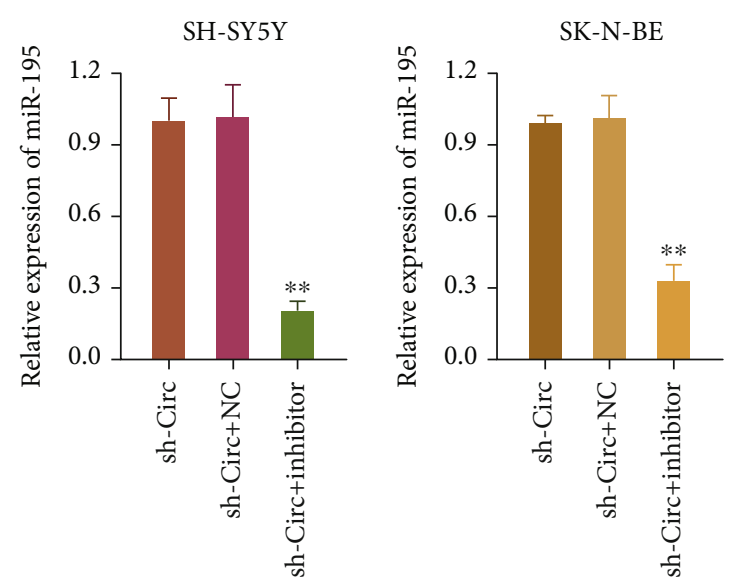

(a)

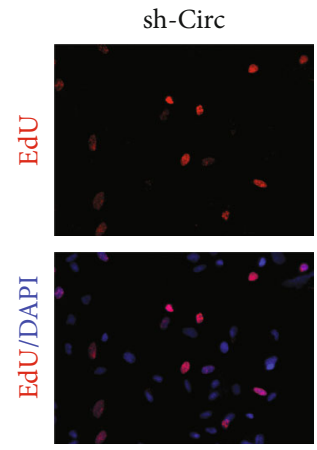

sh-Circ $+\mathrm{NC}$
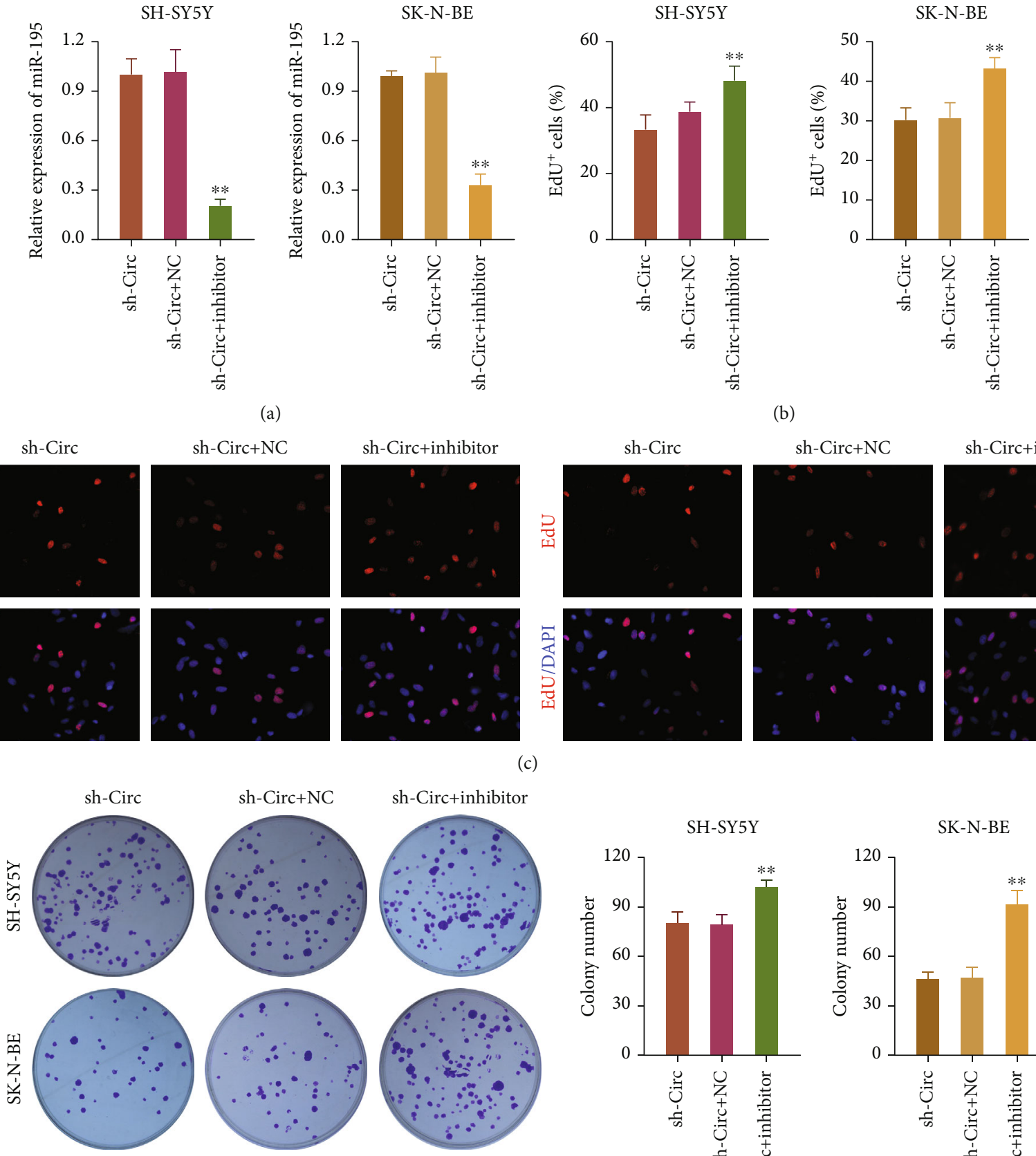

(b)
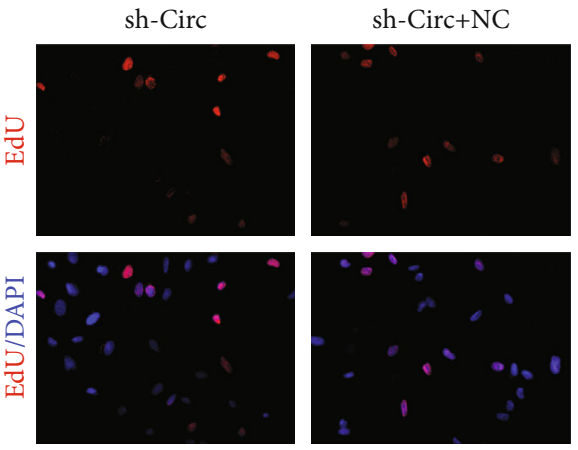

sh-Circ+inhibitor
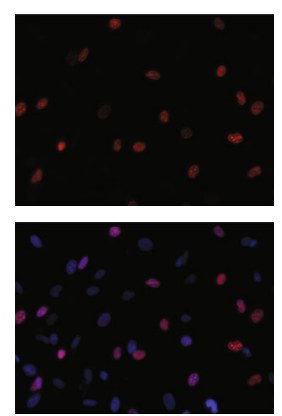

(c)
SH-SY5Y

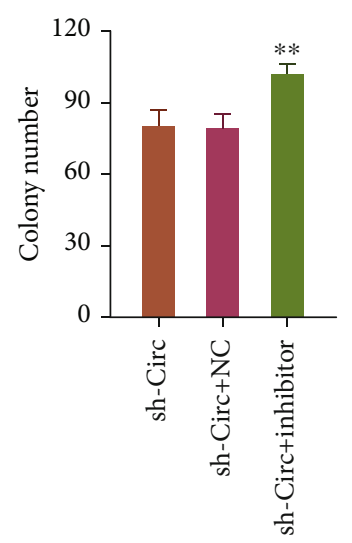

SK-N-BE

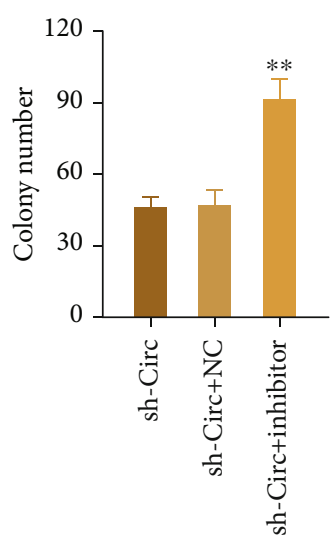

(d)

FIGURE 6: An miR-195 inhibitor reversed the inhibitory effect of circ_0013401 knockdown on NB cell proliferation. (a) circ_0013401silenced SH-SY5Y and SK-N-BE cells were transfected with an miR-195 inhibitor or NC, and miR-195 expression was confirmed by RTqPCR. (b, c) SH-SY5Y and SK-N-BE cells were cotransfected with circ_0013401 shRNA and the miR-195 inhibitor, and cell proliferation was examined by EdU staining. The percentage of $\mathrm{EdU}^{+}$cells was calculated. (d) Clone formation assays were also performed to assess the proliferation of cotransfected SH-SY5Y and SK-N-BE cells. ${ }^{* *} p<0.01$.

and upregulated by circ_0013401 knockdown in SH-SY5Y and SK-N-BE cells $(p<0.05, p<0.01$, Figure $4(\mathrm{c}))$. In contrast, PAK2 could be markedly upregulated by circ 0013401 overexpression and dramatically downregulated by circ_0013401 knockdown in SH-SY5Y and SK-N-BE cells $(p<0.05, p<0.01$, Figure $4(\mathrm{~d}))$. Meanwhile, results of IF studies indicated that circ_0013401 overexpression increased PAK2 expression and reduced LC3B expression; meanwhile, circ_0013401 knockdown significantly decreased PAK2 expression and increased LC3B expression in $\mathrm{SH}$ SY5Y and SK-N-BE cells (Figure 4(e)). More importantly, western blot studies showed that circ_0013401 overexpression 

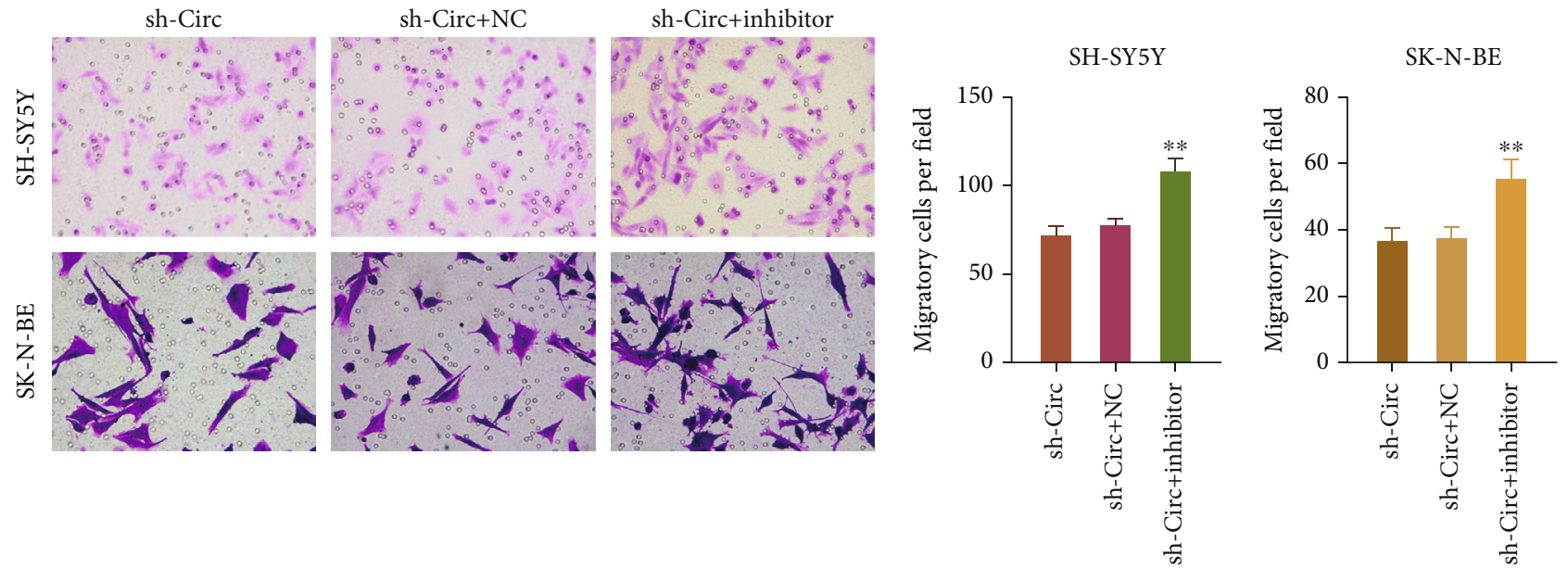

(a)
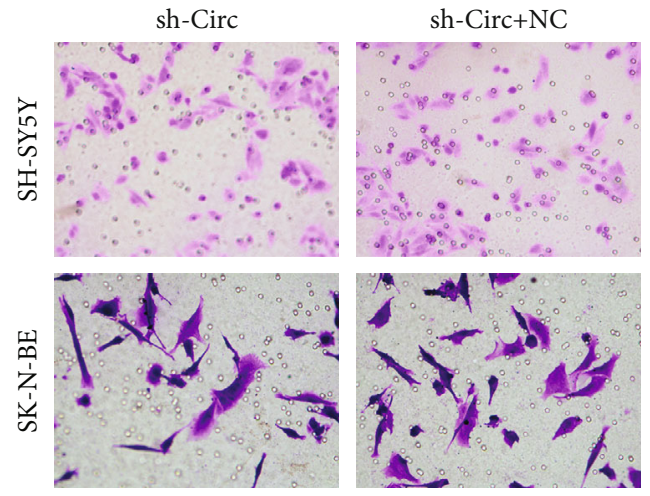

sh-Circ+inhibitor
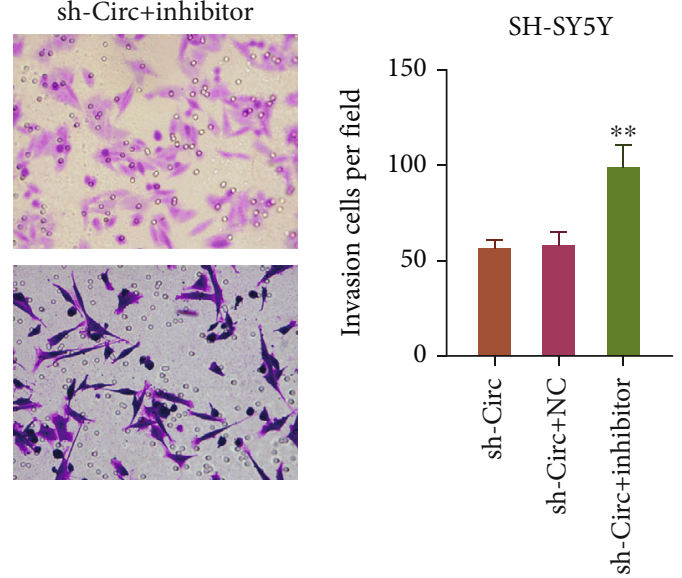

SK-N-BE

(b)
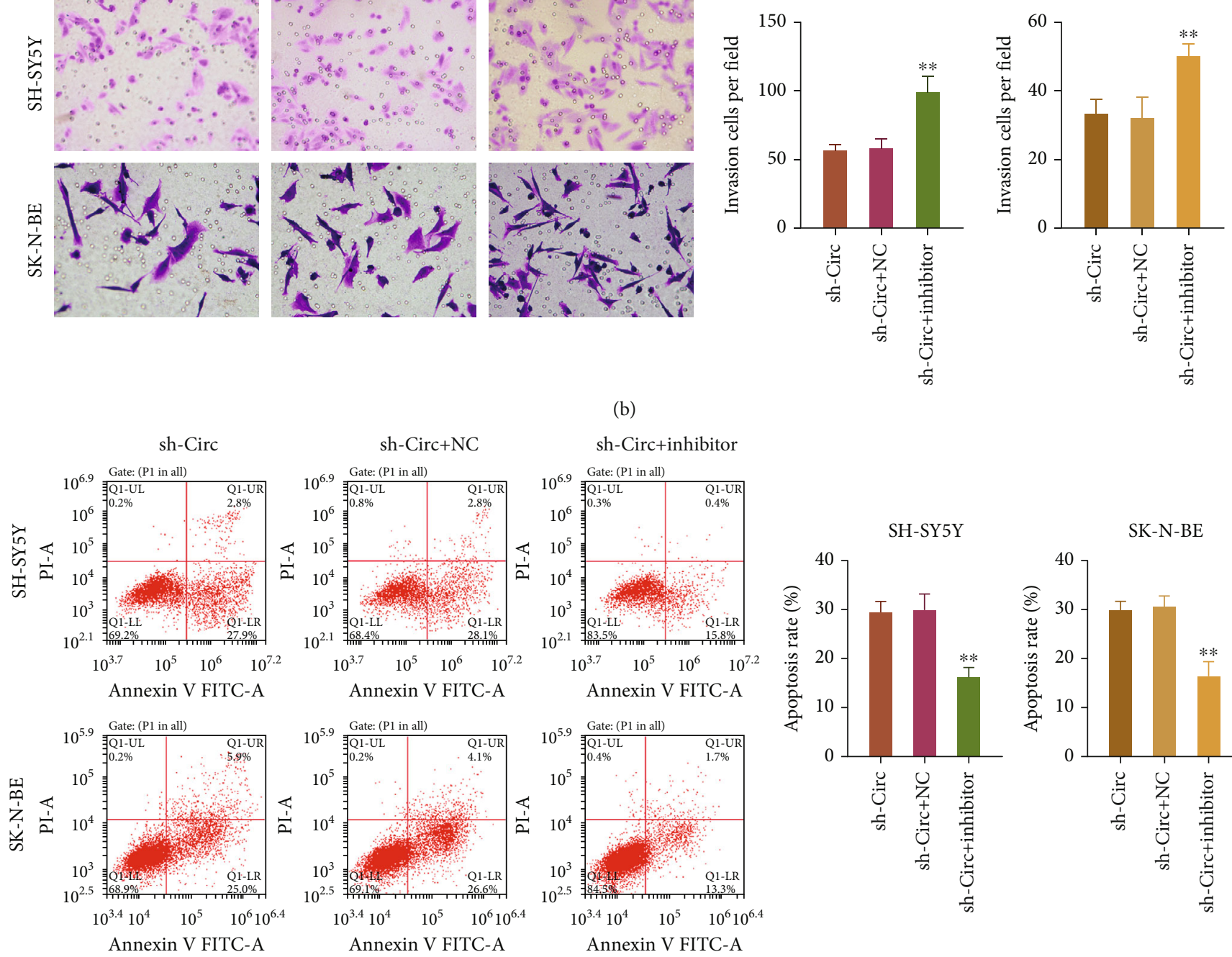

(c)

FIGURE 7: miR-195 was involved in the inhibition of migration and invasion and induction of apoptosis mediated by circ_0013401 knockdown in NB cells. (a, b) The effects of circ_0013401 shRNA and the miR-195 inhibitor on the migration and invasion of SH-SY5Y and SK-N-BE cells were examined by Transwell assays. Magnification, $\times 200$. (c) The apoptosis rates of SH-SY5Y and SK-N-BE cells cotransfected with circ_0013401 shRNA and the miR-195 inhibitor were analyzed by flow cytometry. ${ }^{* *} p<0.01$. 


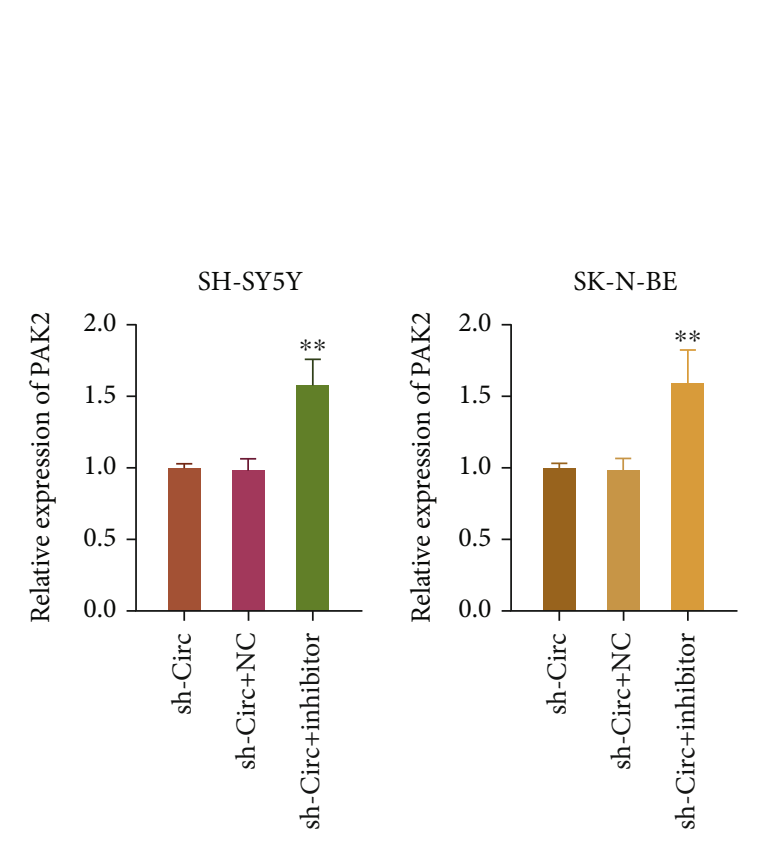

(a)
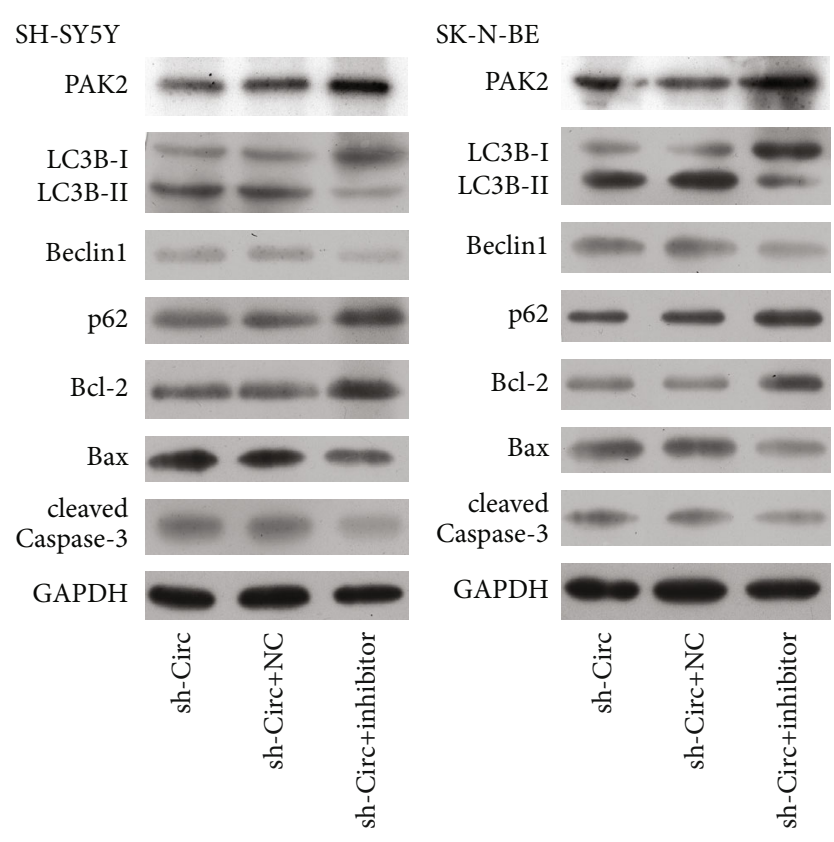

(b)

FIGURE 8: Treatment with an miR-195 inhibitor attenuated the downregulation effects of circ_0013401 knockdown on PAK2 and autophagy- and apoptosis-related proteins in NB cells. (a) The levels of PAK2 in circ_0013401 shRNA and miR-195 inhibitor-transfected SH-SY5Y and SK-N-BE cells were examined by RT-qPCR. (b) The levels of PAK2, LC3B, Beclin1, p62, Bcl-2, Bax, and cleaved caspase-3 protein expression in SH-SY5Y and SK-N-BE cells cotransfected with circ_0013401 shRNA and the miR-195 inhibitor were examined by western blotting. ${ }^{* *} p<0.01$.

could upregulate the levels of PAK2, p62, and Bcl-2 and downregulate the levels of LC3B II/I, Beclin1, Bax, and cleaved caspase-3 in SH-SY5Y and SK-N-BE cells, while circ 0013401 knockdown produced the opposite effects on these proteins in SH-SY5Y and SK-N-BE cells (Figure 4(f)). When taken together, these findings indicated that circ_0013401 dramatically suppressed autophagy and apoptosis and regulated the miR-195/PAK2 axis in NB cells.

3.5. circ_0013401 Sponged miR-195, and PAK2 Was a Target Gene of $m i R-195$. Luciferase reporter assays were performed to verify the relationship between miR-195 and circ 0013401 or PAK2. Our results showed that miR-195 could significantly reduce the luciferase activity of WT-circ 0013401, but not of Mut-circ_0013401 in SH-SY5Y and SK-N-BE cells $(p<0.01$, Figure 5(a)). Similarly, the luciferase activity of WT-PAK2 was significantly reduced by miR-195, while the luciferase activity of Mut-PAK2 was not affected by miR-195 in SH-SY5Y and SK-N-BE cells $(p<0.01$, Figure 5(b)). Thus, we proved that circ_0013401 could regulate the miR-195/PAK2 axis via targeted binding.

3.6. The Inhibitory Effect of circ_0013401 Knockdown on the Proliferation of NB Cells Could Be Reversed by an miR-195 Inhibitor. Next, rescue assays were performed to confirm whether circ_0013401 induced NC proliferation by targeting miR-195. An miR-195 inhibitor was transfected into circ 0013401-silenced SH-SY5Y and SK-N-BE cells, and a subsequent RT-qPCR analysis showed that the miR-195 inhibitor markedly downregulated miR-195 expression, which was induced by circ_0013401 shRNA in SH-SY5Y and SK-NBE cells $(p<0.01$, Figure $6(\mathrm{a}))$. EdU staining results revealed that the proliferation of SH-SY5Y and SK-N-BE cells was significantly increased in the cotransfection groups (miR195 inhibitor plus circ_0013401 shRNA) when compared with proliferation in the circ_0013401 shRNA transfection groups $(p<0.01$, Figures $6(\mathrm{~b})$ and $6(\mathrm{c}))$. Likewise, results of clone formation assays revealed that the miR-195 inhibitor facilitated NB cell proliferation mediated by circ 0013401 knockdown $(p<0.01$, Figure 6(d)). These findings proved that the inhibition of cell proliferation mediated by circ_0013401 shRNA in NB could be significantly reversed by an miR-195 inhibitor.

3.7. miR-195 Was Involved in the Inhibition of Migration and Invasion and Induction of Apoptosis Mediated by circ 0013401 Knockdown in NB Cells. Transwell assays revealed that the migration and invasion abilities of SH-SY5Y and SK-N-BE cells in the cotransfection groups (miR-195 inhibitor plus circ_0013401 shRNA) were markedly higher than those in the circ_0013401 shRNA transfection groups $(p<0.01$, Figures $7(\mathrm{a})$ and $7(\mathrm{~b}))$. Flow cytometry results showed that transfection with the miR-195 inhibitor significantly reduced the apoptosis rates of SH-SY5Y and SK-N-BE cells, which had been induced by miR-491-5p mimics $(p<0.01$, Figure $7(\mathrm{c}))$. In general, our findings verified that circ_0013401 knockdown prevented the migration and invasion and accelerated the apoptosis of NB cells by sponging miR-195. 
SH-SY5Y

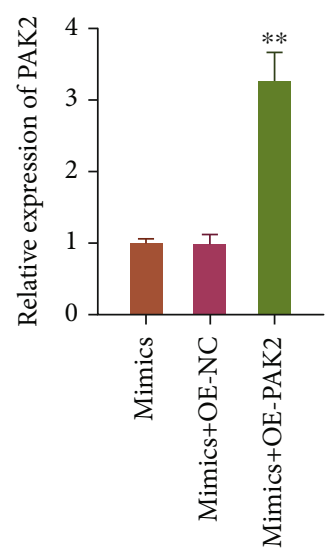

SK-N-BE

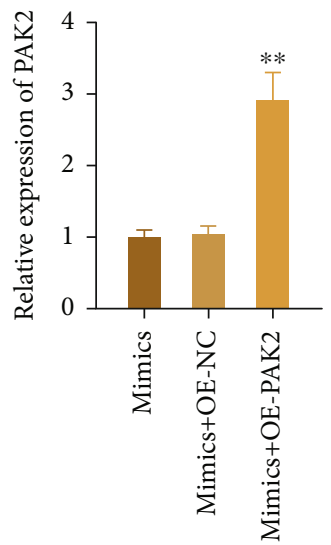

(a)

Mimics

Mimics+OE-NC

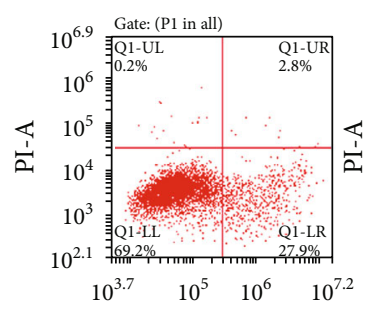

Annexin V FITC-A

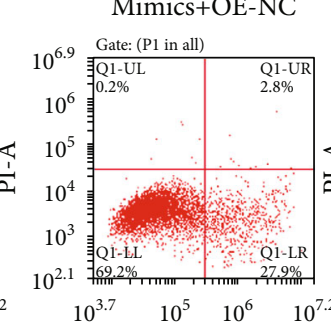

Annexin V FITC-A

SH-SY5Y
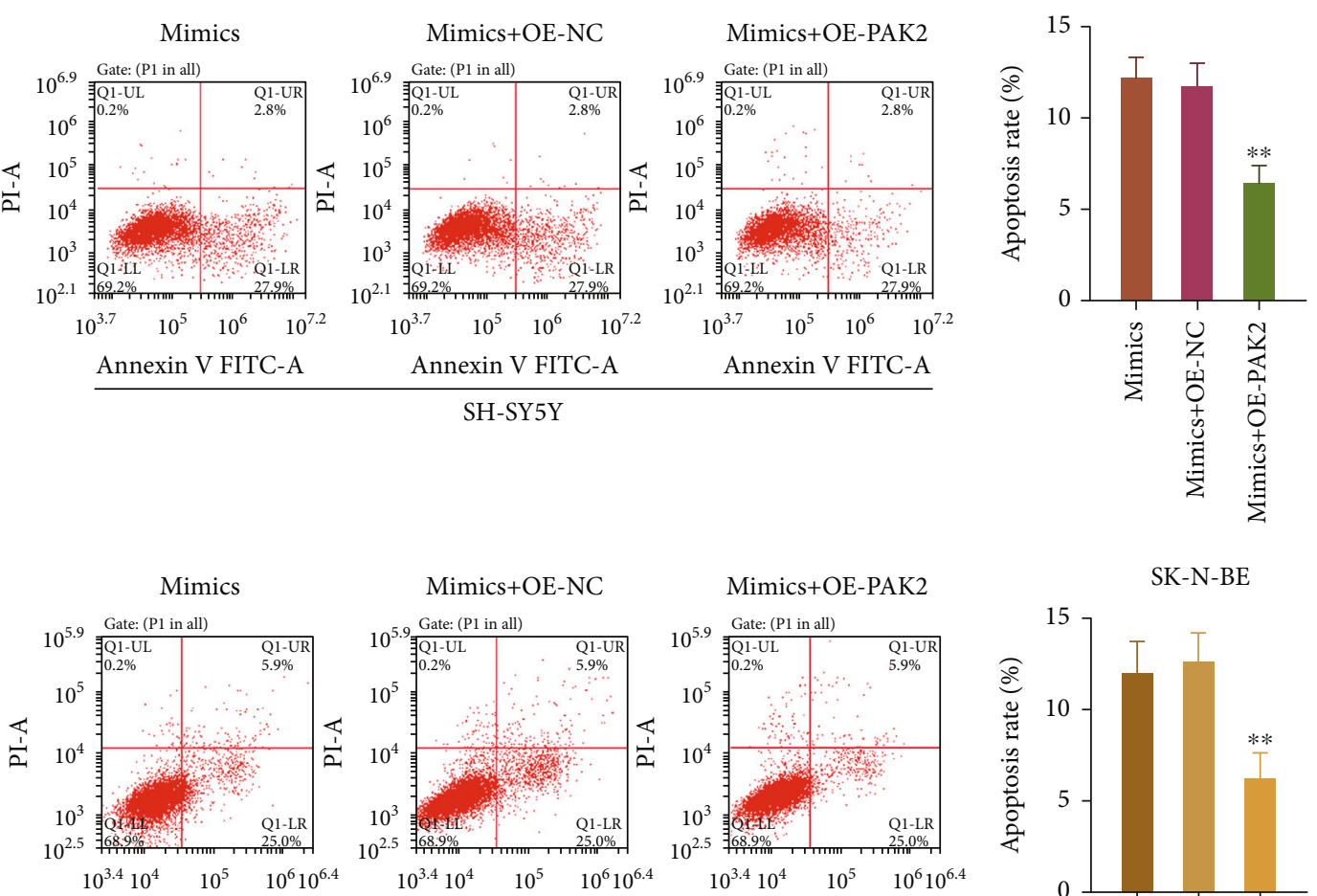

Annexin V FITC-A
Annexin V FITC-A

SK-N-BE

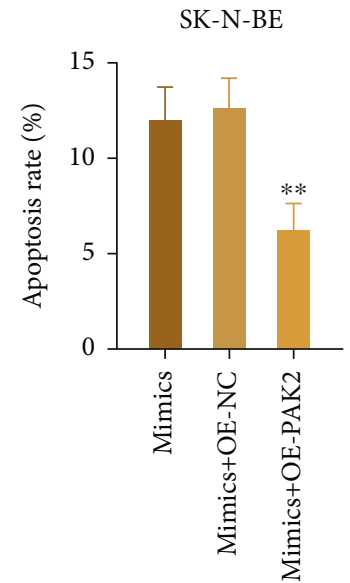

(b)

Figure 9: Continued. 

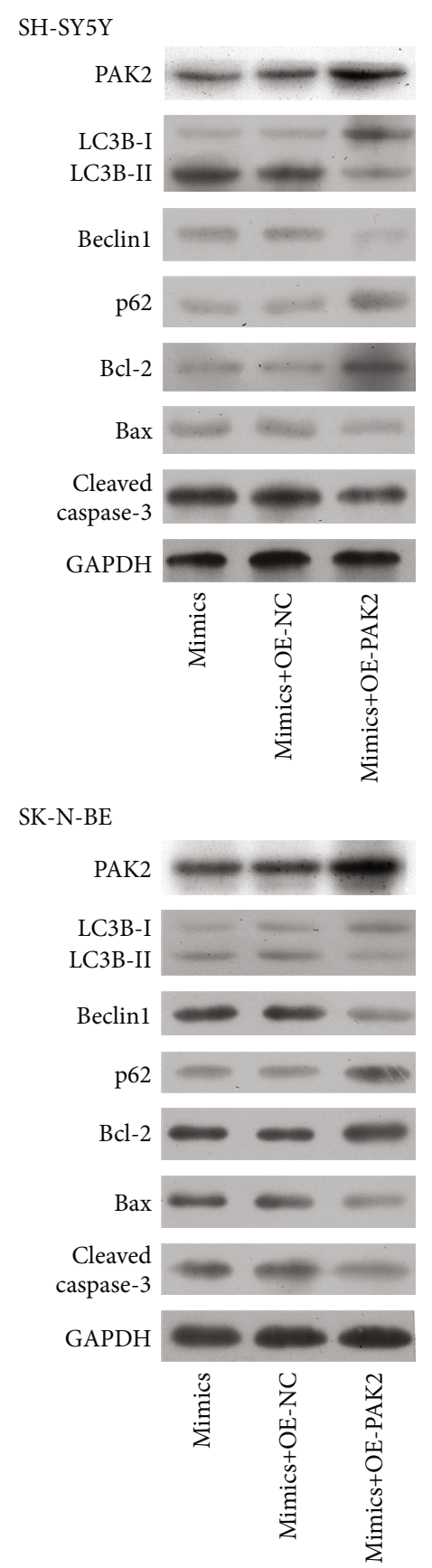

(c)

FIGURE 9: Overexpression of PAK2 suppressed the apoptosis and autophagy mediated by miR-195 in NB cells. SH-SY5Y and SK-N-BE cells were transfected with miR-195 mimics and/or PAK2-overexpression plasmids, respectively. (a) PAK2 expression was assessed by RT-qPCR. (b) Cell apoptosis was assessed by flow cytometry, and the apoptosis rates were calculated. (c) Western blot results for PAK2, LC3B, Beclin1, p62, Bcl-2, Bax, and cleaved caspase-3 proteins. ${ }^{* *} p<0.01$.

3.8. The miR-195 Inhibitor Attenuated the Downregulation Effects of circ_0013401 Knockdown on PAK2 and Autophagy-and Apoptosis-Related Proteins in NB Cells. We next sought to identify downstream molecules that regulated the circ_0013401/miR-195 axis in NB cells. SHSY5Y and SK-N-BE cells were cotransfected with circ_ 0013401 shRNA plus the miR-195 inhibitor; after which, RT-qPCR analyses were performed to determine the levels of PAK2 expression. Those data indicated that PAK2 expression mediated by circ_0013401 knockdown in SHSY5Y and SK-N-BE cells was dramatically upregulated by the miR-195 inhibitor $(p<0.01$, Figure $8(\mathrm{a}))$. Western blot results showed that after transfection with the miR-195 inhibitor, the levels of PAK2, p62, and Bcl-2 expression were upregulated, while the levels of LC3BII/I, Beclin1, Bax, and cleaved caspase- 3 expression were downregulated 


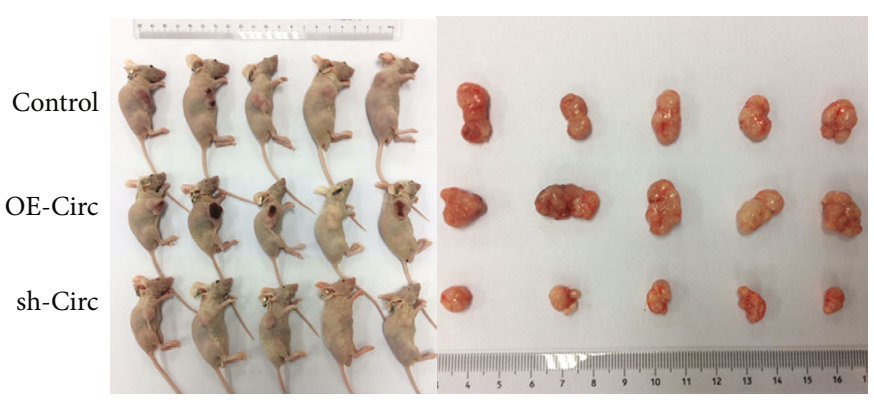

(a)
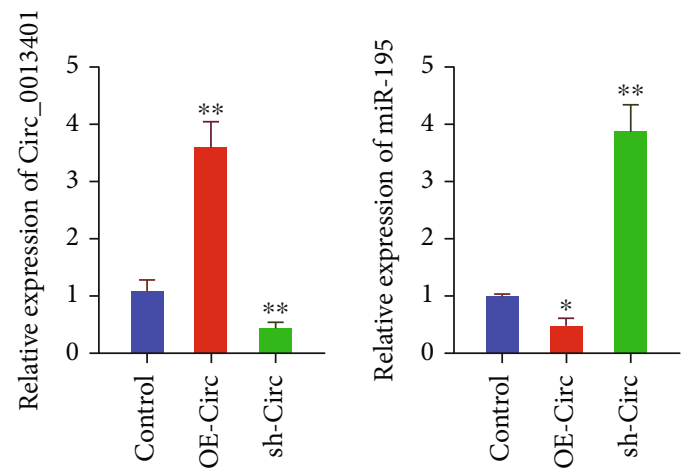

(c)

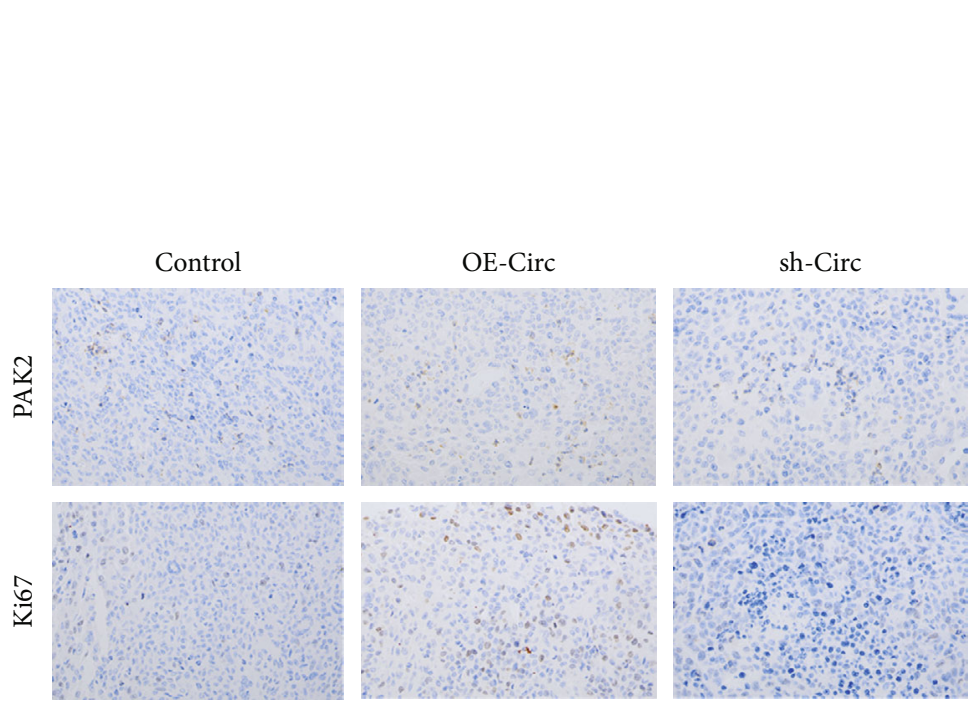

(d)

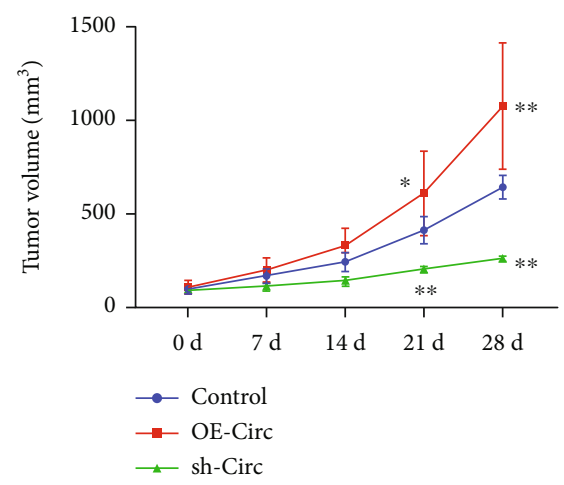

(b)

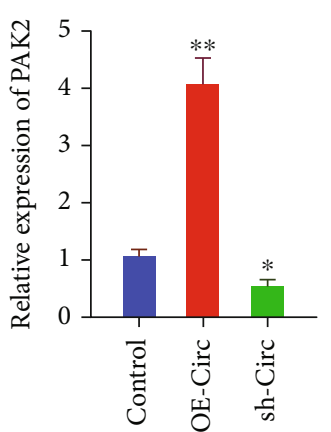

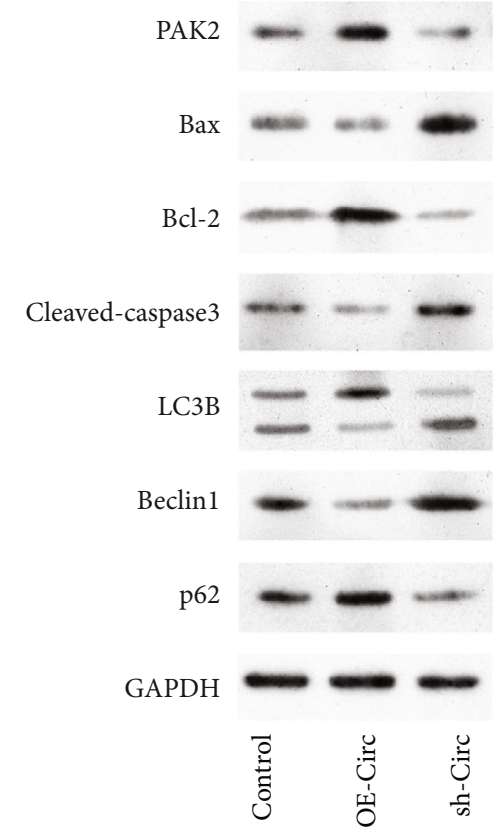

(e)

FIgURE 10: In vivo verification of the circ_0013401/miR-195/PAK2 axis. (a) Nude mice were injected with circ_0013401-overexpressing or circ_0013401-silenced SH-SY5Y cells, and representative images of tumors are shown. (b) Tumor volumes were calculated on days 0, 7, 14, 21, and 28. (c) After circ_0013401 overexpression or knockdown, the levels of circ_0013401, miR-195, and PAK2 expression were examined by RT-qPCR. (d) IHC assays were performed to detect PAK2 and Ki67 expression. (e) The levels of apoptosis- and autophagy-related protein expression were examined by western blotting. ${ }^{*} p<0.05,{ }^{* *} p<0.01$.

in the circ_0013401 shRNA-transfected SH-SY5Y and SK$\mathrm{N}-\mathrm{BE}$ cells (Figure $8(\mathrm{~b})$ ). These results suggested that in NB cells, circ_0013401 knockdown could reduce PAK2 expression and induce autophagy and the synthesis of apoptosis-related proteins by altering the levels of miR195. 


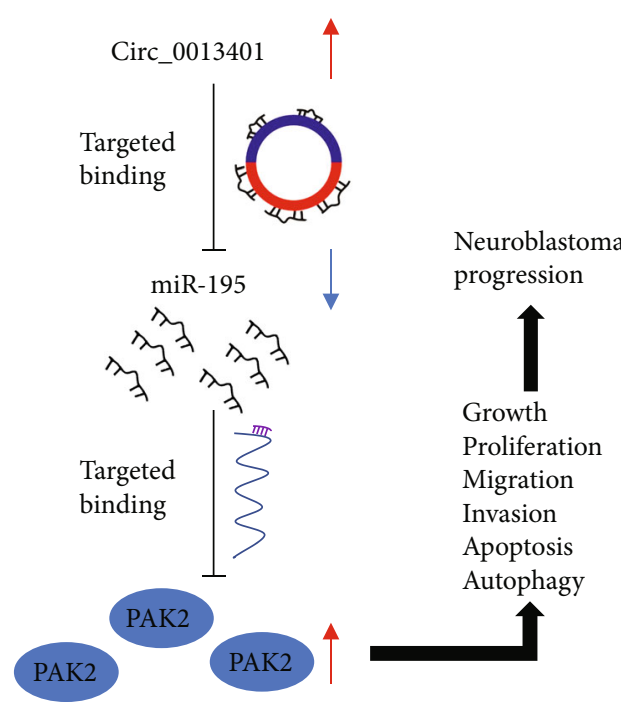

FIGURE 11: A schematic diagram showing how hsa_circ_0013401 functions in NB. Excess circ_0013401 sponges miR-195 and thereby causes an increase in PAK2 expression, which subsequently promotes the growth, proliferation, migration, and invasion, but prevents the apoptosis and autophagy of NB cells.

3.9. Overexpression of PAK2 Suppressed the Apoptosis and Autophagy Mediated by miR-195 in NB Cells. Rescue assays were performed to further verify the effects of the miR195/PAK2 axis on apoptosis and autophagy in NB cells. miR-195 mimics and/or PAK2-overexpression plasmids were transfected into SH-SY5Y and SK-N-BE cells, and PAK2 expression was assessed using the RT-qPCR. As shown in Figure 9(a), overexpression of PAK2 significantly increased PAK2 expression in the miR-195 mimictransfected SH-SY5Y and SK-N-BE cells $(p<0.01)$. Flow cytometry data revealed that the apoptosis rates of those transfected cells were markedly decreased by PAK2 overexpression $(p<0.01$, Figure $9(\mathrm{~b}))$. These findings indicated that overexpression of PAK2 also could significantly increase p62 and Bcl-2 expression and decrease the levels of LC3BII/I, Beclin1, Bax, and cleaved caspase-3 expression in miR-195 mimic-transfected SH-SY5Y and SK-N-BE cells (Figure 9(c)). Overall, the studies showed that PAK2 was significantly involved in the effects of miR-195 on apoptosis and autophagy in NB cells.

3.10. Verification of the circ_0013401/miR-195/PAK2 Axis in an In Vivo Experiment. Based on results of our in vitro experiments, we sought to further verify the regulatory effects of circ_0013401 on NB tumor growth and miR195/PAK2 expression in vivo. We first established cultures of transfected SH-SY5Y cells that either overexpressed circ_0013401 or were silenced and then injected the transfected cells into groups of BALB/c nude mice. After 28 days, the mice were sacrificed and the tumors were removed. We found that NB tumor growth was markedly promoted by circ_0013401 overexpression and suppressed by circ_ 0013401 knockdown $(p<0.01)$ (Figures $10(\mathrm{a})$ and $10(\mathrm{~b})$ ). Additionally, RT-qPCR results revealed that circ_0013401 and PAK2 expression was significantly upregulated and
miR-195 expression was dramatically downregulated in the circ_0013401-overexpression group when compared with the control group. Furthermore, circ_0013401 and PAK2 expression was notably downregulated, and miR-195 expression was significantly upregulated in the circ_0013401silenced group when compared with the control group $(p<0.05, p<0.01$, Figure 10(c)). IHC results also showed that circ_0013401 overexpression notably increased the levels of PAK2 and Ki67 expression, and circ_0013401 knockdown dramatically lowered the levels of PAK2 and Ki67 expression in NB tumor tissues (Figure 10(d)). Moreover, we further verified that overexpression of circ 0013401 markedly upregulated the levels of PAK2, p62, and Bcl-2 expression and downregulated LC3B II/I, Beclin1, Bax, and cleaved caspase- 3 expression in mouse tumor tissues; knockdown of circ_0013401 produced the opposite effects on the expression of all these proteins in mouse tumor tissues (Figure 10(e)). Hence, we further verified that circ_0013401 could significantly downregulate miR-195 and upregulate PAK2 and inhibit apoptosis and the production of autophagy-related proteins in vivo.

\section{Discussion}

NB is the most common extracranial malignant tumor diagnosed in children and accounts for $17 \%$ of all childhood cancer-related deaths [23]. For high-risk NB children, the therapeutic effect of comprehensive treatment is very limited, and adverse reactions can be easily observed [24]. Therefore, there is an urgent need to explore the pathogenesis of $\mathrm{NB}$ and develop new strategies for treating $\mathrm{NB}$ patients. Numerous circRNAs have been reported to play a role in a variety of diseases, especially cancer $[25,26]$. Studies have shown that circRNAs can dramatically affect the biological processes of cancers, including cancer cell differentiation, proliferation, apoptosis, migration, invasion, and autophagy [27, 28]. Due to their specificity and stability, circRNAs have joined miRNAs and lncRNAs in becoming a hotspot for clinical research [29]. However, the roles played by some circRNAs in NB remain largely unclear. In the present study, we used RT-qPCR to examine the expression of circ_0013401, circ_0045997, circ_0077578, and circ_ 0080307 in NB tissues and cells and found that circ_0013401 was dramatically upregulated in NB tissues and cells. Thus, we successfully screened out the potential circRNA (circ 0013401) that can influence NB processes.

Tumor progression is a somatic mutation [30]. Overexpression and silencing of certain genes in tumor cells lead to differences in protein expression from surrounding cells or tissues, and then, tumor cells exhibit different biological behaviors, including excessive proliferation, enhanced migration and invasion capabilities, and apoptosis inhibition $[31,32]$. Current studies on NB also focus on inhibiting proliferation, migration, and invasion and inducing apoptosis of tumor cells [33]. In our study, in vitro and in vivo experiments revealed that hsa_circ_0013401 could induce the proliferation of NB cells and the growth of NB tumors. Moreover, our data also proved that hsa_circ_0013401 could markedly facilitate the migration and invasion of NB cells 
and prevent their apoptosis. Therefore, we indicated that hsa_circ_0013401 could accelerate NB progression.

Autophagy, as a basic physiological phenomenon, is a key mechanism to maintain cell homeostasis [34]. Abnormal regulation of autophagy is relevant to tumor inflammation, energy metabolism, programmed cell death, and drug resistance [35]. Cells can obtain energy during the autophagic process under various cellular stresses, including nutrient depletion, ischemia, and oxidative stress [36]. In tumor cells, autophagy may act as a self-defensive mechanism which contributes to tumor cell survival by removing toxins and garbage [37], while abnormal autophagic activity may cause the inappropriate degradation of cell components that are indispensable for tumor cell survival, resulting in autophagic cell death [38]. Currently, circRNAs have been certified to have a key regulatory effect on tumor cell autophagy [39, 40]. And our research first verified that circ_0013401 could notably repress NB cell autophagy. However, the role of circ_0013401 in NB cell autophagy is not entirely clear, and further study also is needed to validate the change in autophagic flux in NB cells using inhibitors of autophagosomelysosome fusion or autophagolysosome hydrolases.

Based on the theory of ceRNAs, circRNAs could be involved in the tumorigenesis of cancers by functioning as miRNA spongers to regulate the expression of their target mRNAs $[41,42]$. The specific binding of miRNAs has been considered to be an instinctive function of circRNAs in the circRNA-miRNA-mRNA axis [43]. Therefore, we performed a bioinformatics analysis to construct the hypothetical circRNA-miRNA-mRNA networks of circ_0013401. We also proved that circ_0013401 could act as a sponge for miR-195 and verified that miR-195 directly interacted with circ 0013401 and PAK2 in NB cells. Moreover, we showed that circ_0013401 could indirectly regulate PAK2 expression in NB cells by sponging miR-195. Other recent studies have also suggested that miR-195 is closely related to the progression of numerous cancers, including glioma [44], acute myeloid leukemia [45], bladder cancer [46], breast cancer [47], and cervical cancer [48]. In our study, we further demonstrated that an miR-195 inhibitor could reverse the inhibition of cell proliferation, migration, and invasion and induction of cell apoptosis mediated by circ_0013401 knockdown in NB cells. Moreover, we found that circ_0013401 could upregulate PAK2 expression in NB by targeting miR-195.

PAK belongs to the serine/threonine kinase family of proteins, all of which are highly conserved [49]. As a member of the PAK family, PAK is a downstream effector of GTPase in the Rho family [50]. Multiple studies have proven that PAK2 significantly affects cell proliferation, movement, and apoptosis [51, 52]. Abnormal PAK2 function can result in the occurrence of various diseases (including tumors) [50, $53,54]$. A previous study reported that the combination of Rac, Cdc42, and PAK2 could activate PAK2 to induce cell proliferation and growth [55]. Moreover, PAK2 can be hydrolyzed by aspartic and cysteine peptidases and play a role in regulating cell apoptosis [56]. Current studies have verified that PAK2 is overexpressed in a variety of malignant tumor cells, including lung cancer [57], gastric cancer [58], pancreatic cancer [59], and breast cancer [60]. In our study, we further demonstrated that PAK2 was significantly upregulated in NB and that PAK2 expression could be significantly reduced by $\mathrm{miR}-195$ and elevated by circ_0013401 overexpression in NB. Additionally, we found that overexpression of PAK2 could also dramatically suppress the apoptosis and autophagy mediated by miR-195 in NB cells.

\section{Conclusions}

Our findings prove that the circ_0013401/miR-195/PAK2 axis plays crucial roles in $\mathrm{NB}$ progression both in vitro and in vivo (Figure 11). Therefore, our results further increase our understanding of NB pathogenesis and can assist in identifying new therapeutic targets for use in treating NB patients.

\section{Abbreviations}

CCK-8: Cell Counting Kit-8

circRNA: Circular RNA

ceRNA: Competing endogenous RNA

ECL: $\quad$ Enhanced chemiluminescence

FBS: $\quad$ Fetal bovine serum

FISH: Fluorescence in situ hybridization

GN: Gangliocytoma

IHC: Immunohistochemistry

miRNAs: MicroRNAs

miR-195: MicroRNA-195

MREs: miRNA reaction elements

Mut: Mutant

NC: Negative control

NB: Neuroblastoma

OD: Optical density

PAK2: P21-activated kinase 2

RT-PCR: Quantitative real-time PCR

TEM: Transmission electron microscopy

WT: Wild type.

\section{Data Availability}

The datasets supporting the conclusions of this article are included within the article.

\section{Additional Points}

Clinical Perspectives. (1) Neuroblastoma (NB) is a common malignant embryonal solid tumor in children. Circular RNAs (circRNAs) are characterized by a stable structure, high abundance, and tissue-specific expression. Recent studies have revealed that circRNAs play an essential role in regulating gene expression by functioning as competitive endogenous RNAs (ceRNAs). (2) Our current research proves that hsa_circ_0013401 can induce NB progression through miR-195 to enhance PAK2 expression. (3) Our experimental verification of an hsa_circ_0013401/miR195/PAK2 axis in NB lays a foundation for the application of hsa_circ_0013401 and its related regulatory pathways (miR-195/PAK2) in the clinical treatment of NB. 


\section{Ethical Approval}

The study protocol and consent procedures were approved by the Ethics Committee of Guangzhou Medical University (Guangzhou, China).

\section{Consent}

Informed consent was obtained from each patient.

\section{Disclosure}

Please see the link of preprint in https://www.researchsquare .com/article/rs-149430/v1. The funders had no role in the design or conduct of the study; collection, management, analysis, and interpretation of the data; preparation, review, or approval of the manuscript; or the decision to submit the manuscript for publication.

\section{Conflicts of Interest}

The authors declare that they have no conflict of interest.

\section{Authors' Contributions}

Wei Jia and Shibo Zhu were responsible for the conceptualization and funding acquisition, wrote the original draft, and reviewed and edited the manuscript. Xiangliang Tang, Xiaofeng Gao, and Jingqi Zhang were responsible for the resources, data curation, and formal analysis. Yanhong Cui and Dian Li were responsible for the investigation, visualization, methodology, and project administration. All authors read and approved the final manuscript.

\section{Acknowledgments}

This work was supported by grants from the Natural Science Foundation of Guangdong Province of China (No. 2019A1515011178), Guangzhou Institute of Pediatrics/Guangzhou Women and Children's Medical Center (No. GWCMC2020-4-009), and Science and Technology Projects in Guangzhou (No. 202102020097).

\section{References}

[1] K. K. Matthay, J. M. Maris, G. Schleiermacher et al., "Neuroblastoma," Nature Reviews Disease Primers, vol. 2, no. 1, article 16078, 2016.

[2] N. Aygun, "Biological and genetic features of neuroblastoma and their clinical importance," Current Pediatric Reviews, vol. 14, no. 2, pp. 73-90, 2018.

[3] A. Nakagawara, Y. Li, H. Izumi, K. Muramori, H. Inada, and M. Nishi, "Neuroblastoma," Japanese Journal of Clinical Oncology, vol. 48, no. 3, pp. 214-241, 2018.

[4] M. R. Esposito, S. Aveic, A. Seydel, and G. P. Tonini, "Neuroblastoma treatment in the post-genomic era," Journal of Biomedical Science, vol. 24, no. 1, p. 14, 2017.

[5] C. C. Swift, M. J. Eklund, J. M. Kraveka, and A. L. Alazraki, "Updates in diagnosis, management, and treatment of neuroblastoma," Radiographics, vol. 38, no. 2, pp. 566-580, 2018.
[6] S. MacFarland and R. Bagatell, "Advances in neuroblastoma therapy," Current Opinion in Pediatrics, vol. 31, no. 1, pp. 14-20, 2019.

[7] L. S. Kristensen, M. S. Andersen, L. V. W. Stagsted, K. K. Ebbesen, T. B. Hansen, and J. Kjems, "The biogenesis, biology and characterization of circular RNAs," Nature Reviews Genetics, vol. 20, no. 11, pp. 675-691, 2019.

[8] Z. Zhang, T. Yang, and J. Xiao, "Circular RNAs: promising biomarkers for human diseases," eBioMedicine, vol. 34, pp. 267-274, 2018.

[9] L. Szabo and J. Salzman, "Detecting circular RNAs: bioinformatic and experimental challenges," Nature Reviews Genetics, vol. 17, no. 11, pp. 679-692, 2016.

[10] S. P. Barrett and J. Salzman, "Circular RNAs: analysis, expression and potential functions," Development, vol. 143, no. 11, pp. 1838-1847, 2016.

[11] K. Zeng, X. Chen, M. Xu et al., "CircHIPK3 promotes colorectal cancer growth and metastasis by sponging miR-7," Cell Death \& Disease, vol. 9, no. 4, p. 417, 2018.

[12] W. Bi, J. Huang, C. Nie et al., "CircRNA circRNA_102171 promotes papillary thyroid cancer progression through modulating CTNNBIP1-dependent activation of $\beta$-catenin pathway," Journal of Experimental \& Clinical Cancer Research, vol. 37, no. 1, p. 275, 2018.

[13] Q. Lu, T. Liu, H. Feng et al., "Circular RNA circSLC8A1 acts as a sponge of miR-130b/miR-494 in suppressing bladder cancer progression via regulating PTEN," Molecular Cancer, vol. 18, no. 1, p. 111, 2019.

[14] C. Li, M. Li, and Y. Xue, "Downregulation of CircRNA CDR1as specifically triggered low-dose diosbulbin-B induced gastric cancer cell death by regulating miR-7-5p/REG $\gamma$ axis," Biomedicine \& Pharmacotherapy, vol. 120, article 109462, 2019.

[15] L. Xia, M. Song, M. Sun, F. Wang, and C. Yang, "Circular RNAs as biomarkers for cancer," Advances in Experimental Medicine and Biology, vol. 1087, pp. 171-187, 2018.

[16] M. Bhaskaran and M. Mohan, "MicroRNAs: history, biogenesis, and their evolving role in animal development and disease," Veterinary Pathology, vol. 51, no. 4, pp. 759774, 2014.

[17] S. W. Qianqian Tang, X. Qiao, F. Wang, and Y. Wang, "MiR29 promotes ovarian carcinoma cell proliferation through the PTEN pathway," European Journal of Gynaecological Oncology, vol. 41, no. 5, pp. 774-778, 2020.

[18] A. C. Panda, "Circular RNAs act as miRNA sponges," Advances in Experimental Medicine and Biology, vol. 1087, pp. 67-79, 2018.

[19] H. Maroof, S. Irani, A. Arianna, J. Vider, V. Gopalan, and A. K. Y. Lam, "Interactions of vascular endothelial growth factor and p53 with miR-195 in thyroid carcinoma: possible therapeutic targets in aggressive thyroid cancers," Current Cancer Drug Targets, vol. 19, no. 7, pp. 561-570, 2019.

[20] B. Li, S. Wang, and S. Wang, "MiR-195 suppresses colon cancer proliferation and metastasis by targeting WNT3A," Molecular Genetics and Genomics, vol. 293, no. 5, pp. 12451253, 2018.

[21] Y. Jin, M. Wang, H. Hu, Q. Huang, Y. Chen, and G. Wang, "Overcoming stemness and chemoresistance in colorectal cancer through miR-195-5p-modulated inhibition of notch signaling," International Journal of Biological Macromolecules, vol. 117, pp. 445-453, 2018. 
[22] X. Zhu, T. Wu, Y. Chi et al., "MicroRNA-195 suppresses enterovirus A71-induced pyroptosis in human neuroblastoma cells through targeting NLRX1," Virus Research, vol. 292, article 198245, 2021.

[23] I. Kalashnikova, J. Mazar, C. J. Neal et al., "Nanoparticle delivery of curcumin induces cellular hypoxia and ROS-mediated apoptosis via modulation of $\mathrm{Bcl}-2 / \mathrm{Bax}$ in human neuroblastoma," Nanoscale, vol. 9, no. 29, pp. 10375-10387, 2017.

[24] S. B. Whittle, V. Smith, E. Doherty, S. Zhao, S. McCarty, and P. E. Zage, "Overview and recent advances in the treatment of neuroblastoma," Expert Review of Anticancer Therapy, vol. 17, no. 4, pp. 369-386, 2017.

[25] E. Arnaiz, C. Sole, L. Manterola, L. Iparraguirre, D. Otaegui, and C. H. Lawrie, "CircRNAs and cancer: biomarkers and master regulators," Seminars in Cancer Biology, vol. 58, pp. 90-99, 2019.

[26] R. Zhou, Y. Wu, W. Wang et al., "Circular RNAs (circRNAs) in cancer," Cancer Letters, vol. 425, pp. 134-142, 2018.

[27] L. S. Kristensen, T. B. Hansen, M. T. Ven $\varnothing$, and J. Kjems, "Circular RNAs in cancer: opportunities and challenges in the field," Oncogene, vol. 37, no. 5, pp. 555-565, 2018.

[28] J. N. Vo, M. Cieslik, Y. Zhang et al., "The landscape of circular RNA in cancer," Cell, vol. 176, no. 4, pp. 869-881.e13, 2019.

[29] L. J. Li, R. X. Leng, Y. G. Fan, H. F. Pan, and D. Q. Ye, “Translation of noncoding RNAs: focus on lncRNAs, pri-miRNAs, and circRNAs," Experimental Cell Research, vol. 361, no. 1, pp. 1-8, 2017.

[30] J. Skokowa, D. C. Dale, I. P. Touw, C. Zeidler, and K. Welte, "Severe congenital neutropenias," Nature Reviews Disease Primers, vol. 3, no. 1, article 17032, 2017.

[31] I. Pastushenko and C. Blanpain, "EMT transition states during tumor progression and metastasis," Trends in Cell Biology, vol. 29, no. 3, pp. 212-226, 2019.

[32] C. A. Klein, "Cancer progression and the invisible phase of metastatic colonization," Nature Reviews Cancer, vol. 20, no. 11, pp. 681-694, 2020.

[33] M. Schulte, J. Köster, S. Rahmann, and A. Schramm, "Cancer evolution, mutations, and clonal selection in relapse neuroblastoma," Cell and Tissue Research, vol. 372, no. 2, pp. 263268, 2018.

[34] Y. Cao, Y. Luo, J. Zou et al., "Autophagy and its role in gastric cancer," Clinica Chimica Acta, vol. 489, pp. 10-20, 2019.

[35] A. V. Onorati, M. Dyczynski, R. Ojha, and R. K. Amaravadi, "Targeting autophagy in cancer," Cancer, vol. 124, no. 16, pp. 3307-3318, 2018.

[36] P. Wang, B. Z. Shao, Z. Deng, S. Chen, Z. Yue, and C. Y. Miao, "Autophagy in ischemic stroke," Progress in Neurobiology, vol. 163-164, pp. 98-117, 2018.

[37] X. Yang, D. D. Yu, F. Yan et al., "The role of autophagy induced by tumor microenvironment in different cells and stages of cancer," Cell \& Bioscience, vol. 5, no. 1, p. 14, 2015.

[38] B. Levine and G. Kroemer, "Autophagy in the pathogenesis of disease,” Cell, vol. 132, no. 1, pp. 27-42, 2008.

[39] H. Yao, B. Han, Y. Zhang, L. Shen, and R. Huang, "Noncoding RNAs and autophagy," Advances in Experimental Medicine and Biology, vol. 1206, pp. 199-220, 2019.

[40] W. Cui, Q. Dang, C. Chen, W. Yuan, and Z. Sun, "Roles of circRNAs on tumor autophagy," Molecular Therapy Nucleic Acids, vol. 23, pp. 918-929, 2021.
[41] Y. Zhong, Y. du, X. Yang et al., "Circular RNAs function as ceRNAs to regulate and control human cancer progression," Molecular Cancer, vol. 17, no. 1, p. 79, 2018.

[42] J. J. Chan and Y. Tay, "Noncoding RNA:RNA regulatory networks in cancer," International Journal of Molecular Sciences, vol. 19, no. 5, p. 1310, 2018.

[43] M. Cortes-Lopez and P. Miura, "Emerging functions of circular RNAs," The Yale Journal of Biology and Medicine, vol. 89, no. 4, pp. 527-537, 2016.

[44] L. P. Chen, N. N. Zhang, X. Q. Ren, J. He, and Y. Li, "miR103/miR-195/miR-15b regulate SALL4 and inhibit proliferation and migration in glioma," Molecules, vol. 23, no. 11, p. 2938, 2018.

[45] Z. Hong, R. Zhang, and H. Qi, "Diagnostic and prognostic relevance of serum miR-195 in pediatric acute myeloid leukemia," Cancer Biomarkers, vol. 21, no. 2, pp. 269-275, 2018.

[46] K. Yang, H. Tang, M. Ding et al., "Expression of miR-195 and MEK1 in patients with bladder cancer and their relationship to prognosis," International Journal of Clinical and Experimental Pathology, vol. 12, no. 3, pp. 843-850, 2019.

[47] L. Yang, Y. Cai, D. Zhang et al., "miR-195/miR-497 regulate CD274 expression of immune regulatory ligands in triplenegative breast cancer," Journal of Breast Cancer, vol. 21, no. 4, pp. 371-381, 2018.

[48] X. Yang, Z. Yan, H. Yang, H. Ni, L. Zhang, and Y. Wang, "Clinical value of combined detection of miR-1202 and miR195 in early diagnosis of cervical cancer," Oncology Letters, vol. 17, no. 3, pp. 3387-3391, 2019.

[49] R. Kumar, R. Sanawar, X. Li, and F. Li, "Structure, biochemistry, and biology of PAK kinases," Gene, vol. 605, pp. 20-31, 2017.

[50] P. Binder, S. Wang, M. Radu et al., "Pak2 as a novel therapeutic target for cardioprotective endoplasmic reticulum stress response," Circulation Research, vol. 124, no. 5, pp. 696-711, 2019.

[51] W. W. Deng, L. Wu, L. L. Bu et al., "PAK2 promotes migration and proliferation of salivary gland adenoid cystic carcinoma," American Journal of Translational Research, vol. 8, no. 8, pp. 3387-3397, 2016.

[52] J. Huang, A. Huang, A. Poplawski, F. DiPino Jr., J. A. Traugh, and J. Ling, "PAK2 activated by Cdc42 and caspase 3 mediates different cellular responses to oxidative stress-induced apoptosis," Biochimica et Biophysica Acta (BBA) - Molecular Cell Research, vol. 1867, no. 4, article 118645, 2020.

[53] T. Y. Cheng, Y. C. Yang, H. P. Wang et al., "Pyruvate kinase M2 promotes pancreatic ductal adenocarcinoma invasion and metastasis through phosphorylation and stabilization of PAK2 protein," Oncogene, vol. 37, no. 13, pp. 1730-1742, 2018.

[54] B. Nuche-Berenguer, I. Ramos-Álvarez, and R. T. Jensen, “The p21-activated kinase, PAK2, is important in the activation of numerous pancreatic acinar cell signaling cascades and in the onset of early pancreatitis events," Biochimica et Biophysica Acta (BBA)-Molecular Basis of Disease, vol. 1862, no. 6, pp. 1122-1136, 2016.

[55] P. N. Reddy, M. Radu, K. Xu et al., "p21-activated kinase 2 regulates HSPC cytoskeleton, migration, and homing via CDC42 activation and interaction with $\beta$-Pix," Blood, vol. 127 , no. 16 , pp. 1967-1975, 2016.

[56] S. J. Eron, K. Raghupathi, and J. A. Hardy, "Dual site phosphorylation of caspase-7 by PAK2 blocks apoptotic activity 
by two distinct mechanisms," Structure, vol. 25, no. 1, pp. 2739, 2017.

[57] Q. Li, X. Wu, L. Guo, J. Shi, and J. Li, "MicroRNA-7-5p induces cell growth inhibition, cell cycle arrest and apoptosis by targeting PAK2 in non-small cell lung cancer," FEBS Open Bio, vol. 9, no. 11, pp. 1983-1993, 2019.

[58] H. Liu, S. H. Shin, H. Chen et al., "CDK12 and PAK2 as novel therapeutic targets for human gastric cancer," Theranostics, vol. 10, no. 14, pp. 6201-6215, 2020.

[59] G. W. Yao, J. R. Bai, and D. P. Zhang, "P21 activated kinase 2 promotes pancreatic cancer growth and metastasis," Oncology Letters, vol. 17, no. 4, pp. 3709-3718, 2019.

[60] Y. Zhang, L. Wester, J. He et al., "IGF1R signaling drives antiestrogen resistance through PAK2/PIX activation in luminal breast cancer," Oncogene, vol. 37, no. 14, pp. 1869-1884, 2018. 